Stefan Suhr(D)

\title{
Optimal results in Lorentzian Aubry-Mather theory
}

Received: 18 June 2020 / Accepted: 18 November 2020 /

Published online: 30 November 2020

Abstract. This article complements the Lorentzian Aubry-Mather Theory in Suhr (Geom Dedicata 160:91-117, 2012; J Fixed Point Theory Appl 21:71, 2019) by giving optimal multiplicity results for the number of maximal invariant measures. As an application the optimal Lipschitz continuity of the time separation on the Abelian cover is established.

\section{Introduction}

The structure of recurrent sets is central to the theory of dynamical systems. For geodesic flows in the absence of curvature or symmetry assumptions or other Tonelli Lagrangian flows the two main methods of producing recurrent sets are via closed orbits and minimal invariant measures in Aubry-Mather theory. In the example of flat tori every invariant set of the geodesic flow is a union of sets of this sort. For compact manifolds both closed orbits and minimal invariant measures are known to exist. In the case of pseudo-Riemannian metrics the situation is different. The tangent bundle outside the zero section trisects into parts with positive, vanishing and negative energy. It is a-priori not clear whether the sectors of positive and negative energy contain any recurrent subsets. In fact for the Clifton-Pohl tori the only recurrent subsets are two closed orbits, see [13]. In general it is not know if every compact pseudo-Riemannian manifold admits a closed geodesic. For a large class of Lorentzian 2-tori it is known though, that they admit many variationally characterized recurrent subsets outside the lightcones, see [10,14,15,17]. These subsets are the support of maximal invariant measures studied in Aubry-Mather theory. The Aubry-Mather theory in [21] gave existence of such invariant measures for class A spacetimes of any dimension. Due to convex geometric considerations the number of distinct maximal invariant measures on a class A spacetime should be bounded below by the first Betti number $b$ of the underlying manifold. The Lorentzian Hedlund examples in [21] realize this bound. Up to this point a general argument was lacking to insure the right multiplicity. In the Riemannian case the lower bound on the number of minimal invariant measures is due to [1].

Stefan Suhr is supported by the SFB/TRR 191 "Symplectic Structures in Geometry, Algebra and Dynamics", funded by the Deutsche Forschungsgemeinschaft

Fakultät für Mathematik, Universität Bochum, Bochum, Germany

e-mail: Stefan.Suhr@ruhr-uni-bochum.de

Mathematics Subject Classification: 37J50 · 53C22 · 53C50

https://doi.org/10.1007/s00229-020-01267-2 
In the present article this is done for class $\mathrm{A}_{1}$ spacetimes, a subclass of class $\mathrm{A}$ spacetimes, into which all known examples fall. See Sect. 2.2 for the definition. The main technical result is Theorem 2.11 showing support functions of the stable time separation are contained in the interior of the dual stable time cone, see Sect. 2.2 for definitions. With this preparation we show in Theorem 2.15 that there are at least $b$-many maximal invariant measures supported entirely in the timelike vectors, i.e. bounded away from the lightcones. If the stable time separation vanishes somewhere outside 0 then there exist infinitely many ergodic maximal probability measures supported entirely in the timelike tangent vectors by Corollary 2.16, generalizing the results of [18]. In Theorem 2.17 the method is applied to show the Lipschitzcontinuity of the time separation of the Abelian cover of a class $A_{1}$ spacetime. See [6] for a related result.

The text is organized as follows. In Sect. 2.1 we introduce the class of uniformly vicious spacetimes and give a smoothing result for the required family of timelike loops (Proposition 2.6). In Sect. 2.2 we then introduce class $A_{1}$ spacetimes together with the necessary prerequisites from the Aubry-Mather theory for class A spacetimes. The main results are discussed in Sect. 2.2. Proofs are then provided in Sect. 3.

Notation. Fix a complete Riemannian metric $g_{R}$ on $M$.

\section{Results}

\subsection{Uniformly vicious spacetimes}

A Lorentzian manifold is a pair $(M, g)$ consisting of a smooth connected manifold $M$ and a smooth tensor field $g \in \Gamma\left(T_{2}^{0} M\right)$ such that $g_{p}$ is a symmetric and nondegenerate bilinear form of index 1, i.e. $g_{p} \cong \operatorname{diag}(-1,1, \ldots, 1)$, for all $p \in M$. For details about Lorentzian geometry refer to the standard textbook references [8], [13] and [2]. For more recent developments in causality theory see [12].

A smooth curve $\gamma: I \rightarrow M$ in a Lorentzian manifold $(M, g)$ is timelike if $g(\dot{\gamma}(t), \dot{\gamma}(t))<0$ for all $t \in I$. For an open subset $U \subseteq M$ define $I_{U} \subseteq U \times U$ to be the set of pairs $(p, q) \in U \times U$ such that there exists a timelike curve $\eta: I \rightarrow U$ with endpoints $p$ and $q$.

Definition 2.1. Let $(M, g)$ be a Lorentzian manifold. A Lipschitz continuous curve $\gamma: I \rightarrow M$ is called essentially timelike if for each $t \in I$ there exist $\delta>0$ and a convex normal neighborhood $U$ of $\gamma(t)$ with $\gamma((t-\delta, t+\delta)) \subseteq U$ such that $(\gamma(\sigma), \gamma(\tau)) \in I_{U}$ for all $\sigma, \tau \in(t-\delta, t+\delta)$.

A loop $\gamma: S^{1} \rightarrow M$ is essentially timelike if a lift $\bar{\gamma}: \mathbb{R} \rightarrow M$ is essentially timelike.

Note that every timelike curve is essentially timelike and every essentially timelike curve $\gamma: I \rightarrow M$ is causal, see Sect. 2.2. It is further easy to see, by example in Minkowski space, that Lipschitz continuous and essentially timelike curves do not necessarily have timelike tangents anywhere.

The definition of essential timelikeness is motivated by the observation following from [16] that every essentially timelike curve can be deformed, with fixed 
endpoints, into a timelike curve via essentially timelike curves. The same is true for essentially timelike loops.

Recall that a Lorentzian manifold $(M, g)$ is (totally) vicious if every point lies on a timelike loop. We can characterize viciousness also in the present terms, see [12].

Fact 2.2. A Lorentzian manifold $(M, g)$ is vicious if and only if every point lies on an essentially timelike loop.

For the subsequent results we will need to strengthen the assumption of viciousness.

Definition 2.3. A Lorentzian manifold $(M, g)$ is uniformly vicious if there exists a smooth connected manifold $\mathcal{M}$ and a continuous proper map $H: \mathcal{M} \times S^{1} \rightarrow M$ such that $\left.H\right|_{\mathcal{M} \times\{0\}}$ is a smooth surjective submersion and the loops $\left.H\right|_{\{x\} \times S^{1}}$ are essentially timelike for all $x \in \mathcal{M}$. We will call the map $H$ a uniform family.

With a uniform family we can choose a continuous family of essentially timelike loops along every subset of $M$.

Remark 2.4. Every uniformly vicious Lorentzian manifold is vicious.

Examples of uniformly vicious Lorentzian manifolds include flat tori or more generally spaces of the form

$$
\left.(M, g)=\left(N \times S^{1},-f^{2} d t^{2}+\beta d t+h\right)\right),
$$

where $f$ is any positive smooth function on $N \times S^{1}, \beta$ is a 1-form on $N$ and $h$ is Riemannian metric on $N$, both depending smoothly on the $S^{1}$-coordinate. Another set of example is provided by any Lorentzian metric on $S^{2 n+1}$ such that the Hopf fibration is timelike.

Proposition 2.5. Assume that $M$ is diffeomorphic either to $T^{2}$, the Klein bottle or $S^{1} \times \mathbb{R}$. Then every vicious Lorentzian metric on $M$ is uniformly vicious.

The proof will be given in Sect. 3.1.

It is easy to construct examples of vicious Lorentzian manifolds that are not uniformly vicious. Consider the quotient of Minkowski space $\left(\mathbb{R}^{2}, d x^{2}-d y^{2}\right)$ by the group of translations $\Gamma:=\mathbb{Z} \cdot(0,1)$. Remove from the quotient the point $[(0,0)]$. The claim is then that $M:=\mathbb{R}^{2} / \Gamma \backslash\{[(0,0)]\}$ together with the induced Lorentzian metric $g$ is vicious, but not uniformly vicious. The viciousness is obvious, since $\left(\mathbb{R}^{2} / \Gamma, d x^{2}-d y^{2}\right)$ is vicious. The other part in the claim assume to the contrary that $(M, g)$ is uniformly vicious. Note that all loops in the uniform family represent the same fundamental class in $\pi_{1}(M)$ since $\mathcal{M}$ is assumed to be connected. In the covering space $\mathbb{R}^{2} \backslash \mathbb{Z} \cdot(0,1)$ the lifts of loops in the uniform family are essentially timelike curves connecting a point $p=\left(p_{1}, p_{2}\right) \in \mathbb{R}^{2} \backslash \mathbb{Z} \cdot(0,1)$ with $p+(0, k)$ for some $k \in \mathbb{Z}$. If $p_{1}>|k|$ it is obvious that no causal curve connecting $p$ with $p+(0, k)$ can intersect the halfspace $\{(x, y) \mid x \leq 0\}$. Therefore the fundamental class of each loop in the uniform family must belong to the subgroup $\left\{a^{n} \mid n \in \mathbb{Z}\right\}$, where $a$ is the fundamental class represented by the projections to $M$ of $t \mapsto$ 
$(x, t) \in \mathbb{R}^{2} \backslash \mathbb{Z} \cdot(0,1), t \in[0,1]$ and $x>0$. The same argument with $p_{1}<-|k|$ shows that the fundamental class of the loops in the uniform family must belong to the subgroup $\left\{b^{n} \mid n \in \mathbb{Z}\right\}$ generated by the the projections to $M$ of the curves $t \mapsto(x, t) \in \mathbb{R}^{2} \backslash \mathbb{Z} \cdot(0,1), t \in[0,1]$ with $x<0$. Since the fundamental group of $M$ is the free group generated by $a$ and $b$, we obtain $k=0$. But this is clearly a contradiction, since Minkowski space does not contain any causal loops.

Even though the existence of a uniform family is easier to verify for essentially timelike loops, we will need the following smooth version in the subsequent discussion. The proof will be given in Sect. 3.1.

Proposition 2.6. Let $(M, g)$ be a uniformly vicious Lorentzian manifold. Then there exists a smooth uniform family $\bar{H}: \mathcal{M} \times S^{1} \rightarrow M$ such that $\left.\bar{H}\right|_{\{x\} \times S^{1}}$ is a timelike loop for every $x \in \mathcal{M}$.

\subsection{Class $A_{1}$ spacetimes}

A Lorentzian manifold $(M, g)$ is time-orientable if the set $\{v \in T M \mid g(v, v)<0\}$ of timelike tangent vectors has exactly two connected components. A choice of either component is a time-orientation. Recall that every Lorentzian manifold has a two-fold time-orientable cover. A time-oriented Lorentzian manifold is a spacetime. A causal tangent vector, i.e. $v \in T M$ with $v \neq 0$ and $g(v, v) \leq 0$, is future pointing if it lies in the component chosen by time-orientation. Otherwise the causal tangent vector is past pointing. A Lipschitz-continuous curve $\gamma: I \rightarrow M$ is future pointing if $\dot{\gamma}$ is future pointing for almost all $t \in I$. A curve $\eta: I \rightarrow M$ is past pointing if its reverse parametrization $\gamma(t):=\eta(-t)$ is future pointing. A curve $\eta$ in a Lorentzian manifold, not necessarily time-oriented, is causal if a lift to a time-orientated cover is future or past pointing. Note that this definition is independent of the lift and the chosen time-orientation.

For a manifold $M$ denote with $\bar{M}$ the Abelian cover, i.e.

$$
\bar{M}=\tilde{M} /\left[\pi_{1}(M), \pi_{1}(M)\right],
$$

where $\tilde{M}$ is the universal cover of $M$.

Definition 2.7. A compact spacetime $(M, g)$ is of class $\mathbf{A}_{1}$ if it is uniformly vicious and the Abelian cover is globally hyperbolic.

It follows directly from the definition that any class $A_{1}$ spacetime is class $A$, see [20]. Recall that a spacetime is globally hyperbolic if it does not contain any causal loops and for all pairs $(p, q) \in M \times M$ the set

$$
\{r \in M \mid r \text { lies on a future pointing curve from } \mathrm{p} \text { to } q\}
$$

is compact, see [3].

Note that in the case of compact uniformly vicious spacetimes the domain $\mathcal{M}$ of the uniform family is compact.

Proposition 2.8. The set of class $A_{1}$ metrics is open in the space of Lorentzian metrics. 
Proof. Every uniformly vicious spacetime admits a smooth uniform family by Proposition 2.6. The existence of a smooth uniform family is obviously an open condition.

Remark 2.9. Following Proposition 2.6 we will always assume that the uniform family of a Class $\mathrm{A}_{1}$ spacetime is smooth.

In order to state the main results we need to recollect some definitions and results from $[11,20,21]$.

For a compact manifold $M$ denote by

$$
b:=\operatorname{dim}_{\mathbb{R}} H_{1}(M, \mathbb{R})
$$

the first Betti number of $M$. Consider a basis $\left\{k_{1}, \ldots, k_{b}\right\}$ of $H_{1}(M, \mathbb{R})$ consisting of integer classes. Denote with $\left\{\alpha_{1}, \ldots, \alpha_{b}\right\}$ the dual basis of $H^{1}(M, \mathbb{R})$ and choose representatives $\omega_{i} \in \alpha_{i}$. For two points $x, y \in \bar{M}$ define $y-x \in H_{1}(M, \mathbb{R})$ through

$$
\left\langle\alpha_{i}, y-x\right\rangle:=\int_{\bar{\gamma}} \bar{\omega}_{i}
$$

where $\bar{\gamma}$ is any Lipschitz curve connecting $x$ and $y$, and $\bar{\omega}_{i}$ is the lift of $\omega_{i}$ to $\bar{M}$. For a curve $\gamma:[a, b] \rightarrow M$ we define

$$
\left\langle\alpha_{i}, \gamma(b)-\gamma(a)\right\rangle:=\int_{\gamma} \omega_{i}
$$

For a compact spacetime $(M, g)$ consider sequences $\gamma_{n}:\left[a_{n}, b_{n}\right] \rightarrow M$ of future pointing curves such that $L^{g_{R}}\left(\gamma_{n}\right) \rightarrow \infty$. Define $\mathfrak{T}^{1} \subseteq H_{1}(M, \mathbb{R})$ to be the set of accumulation points of sequences

$$
\left\{\frac{\gamma_{n}\left(b_{n}\right)-\gamma_{n}\left(a_{n}\right)}{L^{g_{R}}\left(\gamma_{n}\right)}\right\}_{n \in \mathbb{N}} .
$$

The stable timecone $\mathfrak{T}$ of $(M, g)$ then is the cone over $\mathfrak{T}^{1}$. The dual stable time cone is

$$
\mathfrak{T}^{*}:=\left\{\alpha \in H^{1}(M, \mathbb{R})|\alpha|_{\mathfrak{T}} \geq 0\right\} .
$$

Let $\|$.$\| denote the stable norm of \left(M, g_{R}\right)$ on $H_{1}(M, \mathbb{R})$, see [7]. Define

$$
\mathfrak{T}_{\varepsilon}:=\left\{v \in \mathfrak{T} \mid \operatorname{dist}_{\|.\|}(v, \partial \mathfrak{T}) \geq \varepsilon\|v\|\right\}
$$

for $\varepsilon>0$, where dist $\|_{\|.\|}$is the distance function w.r.t. to the stable norm.

The time separation $d: M \times M \rightarrow[0, \infty]$ of a spacetime $(M, g)$ is defined by

$$
d(p, q):=\sup \left\{L^{g}(\gamma) \mid \gamma \text { future pointing from } p \text { to } q\right\},
$$

where

$$
L^{g}(\gamma):=\int_{I} \sqrt{|g(\dot{\gamma}(t), \dot{\gamma}(t))|} d t
$$


denotes the length functional w.r.t $g$. Here the convention $\sup \emptyset:=0$ applies. By the Avez-Seifert Theorem every pair of points in a globally hyperbolic spacetime, connectable by a future pointing curve, is connected by a causal curve realizing the supremum. Further the time separation is continuous for globally hyperbolic spacetimes, see [2].

Recall from [21] the analogue of the stable norm for class A spacetimes:

Theorem 2.10. [21] Let $(M, g)$ be of class A. Then there exists a unique concave positive 1-homogenous function, called the stable time separation,

$$
\mathfrak{l}: \mathfrak{T} \rightarrow \mathbb{R}
$$

such that for every $\varepsilon>0$ there is a constant $\bar{C}(\varepsilon)<\infty$ with

$$
|\mathfrak{l}(y-x)-d(x, y)| \leq \bar{C}(\varepsilon)
$$

for all $x, y \in \bar{M}$ with $y-x \in \mathfrak{T}_{\varepsilon}$.

The stable time separation gives a similar "broad" view onto the time separation of the (globally hyperbolic) Albelian cover as does the stable norm for the distance function of a lifted Riemannian metric. The concavity $l$ corresponds to the convexity of a norm. The most striking difference to the Riemannian case, though, is the boundary of $\mathfrak{T}$ and the behavior of $\mathfrak{l}$ near it. In the simplest examples of class $\mathrm{A}_{1}$ spacetimes, e.g. conformally flat tori, see [18], one observes $l$ to be "anti-Lipschitz" near $\partial \mathfrak{T}$, i.e. the slope of any support function of $\mathfrak{l}$ becoming unbounded. In the case of class $A_{1}$ spacetimes we can confirm this observation with the following result. The proof will be given in Sect. 3.2.

Theorem 2.11. Let $(M, g)$ be of class $A_{1}$. Then every support function $\alpha \in$ $H^{1}(M, \mathbb{R})$ of $\mathfrak{l}$ is contained in $\left(\mathfrak{T}^{*}\right)^{\circ}$.

In order to study timelike geodesics of class $\mathrm{A}_{1}$ spacetimes and their limits in the fashion of Aubry-Mather theory we reparametrize the geodesic flow to be complete, see [21] for the construction. This does not affect our study of the length functional, as it is parametrization invariant.

Definition 2.12. Let $(M, g)$ be a pseudo-Riemannian manifold and $g_{R}$ a complete Riemannian metric on $M$. The pregeodesic flow

$$
\Phi: T M \times \mathbb{R} \rightarrow T M
$$

of $(M, g)$ (relative to $\left.g_{R}\right)$ is the the reparametrization of the geodesic flow of $(M, g)$ w.r.t. constant $g_{R}$-arclength.

Note that the pregeodesic flow is still a conservative flow, i.e. it is defined by a differential equation of second order on $M$. If not noted otherwise pregeodesics are always assumed to be parametrized by $g_{R}$-arclength.

Using the properties of the pregeodesic flow we define the rotation classes $\rho(\mu) \in H_{1}(M, \mathbb{R})$ of finite $\Phi$-invariant Borel measures $\mu$ on on the unit tangent bundle $T^{1} M$ of $\left(M, g_{R}\right)$ by the condition

$$
\langle\alpha, \rho(\mu)\rangle:=\int_{T^{1} M} \omega d \mu
$$


where $\omega$ represents $\alpha \in H^{1}(M, \mathbb{R})$, see [21].

For the obvious reasons we restrict all considerations to measures supported in the future pointing causal vectors. For class A spacetimes it follows that the set of rotation classes of finite invariant measures is $\mathfrak{T}$ and

$$
\mathfrak{l}(h)=\max \left\{\int_{\operatorname{Time}(M, g)} \sqrt{|g(v, v)|} d \mu(v) \mid \rho(\mu)=h\right\}
$$

for all $h \in \mathfrak{T}$, where

$$
\operatorname{Time}(M, g):=\{v \in T M \mid v \text { future pointing timelike }\},
$$

see [21]. An invariant measure $\mu$ with

$$
\mathfrak{l}(\rho(\mu))=\int_{\operatorname{Time}(M, g)} \sqrt{|g(v, v)|} d \mu(v)
$$

is a maximal measure.

Denote with

$$
\operatorname{Light}(M, g)
$$

the set of future pointing lightlike vectors, i.e. causal and non-timelike vectors. Note that both $\operatorname{Time}(M, g)$ and $\operatorname{Light}(M, g)$ are smooth fiber bundles over $M$ with fiber $\operatorname{Time}(M, g)_{p}$ and $\operatorname{Light}(M, g)_{p}$, resp.

Proposition 2.13. Let $(M, g)$ be of class $A_{1}$. Then for every $\varepsilon>0$ there exists $\delta(\varepsilon)>0$ such that

$$
\operatorname{dist}(\operatorname{supp} \mu, \operatorname{Light}(M, g)) \geq \delta(\varepsilon)
$$

for every maximal measure $\mu$ with $\rho(\mu) \in \mathfrak{T}_{\varepsilon}$.

The proof will be given in Sect. 3.2.

We say that a future pointing pregeodesic $\gamma: \mathbb{R} \rightarrow M$, i.e. $\dot{\gamma}$ is future pointing, is a maximizer if one (hence any) lift to $\bar{M}$ is maximal, i.e.

$$
L^{\bar{g}}\left(\left.\bar{\gamma}\right|_{[s, t]}\right)=d(\bar{\gamma}(s), \bar{\gamma}(t))
$$

for all $s \leq t \in \mathbb{R}$.

We say that a finite Borel measure $\mu$ on $T^{1} M$ is a limit measure of the future pointing pregeodesic $\gamma: \mathbb{R} \rightarrow M$ if there exists a sequence of intervals $\left[a_{n}, b_{n}\right]$ with $b_{n}-a_{n} \rightarrow \infty$ and a constant $C \in(0, \infty)$ such that

$$
\frac{C}{b_{n}-a_{n}}(\dot{\gamma})_{\sharp}\left(\left.\mathcal{L}^{1}\right|_{\left[a_{n}, b_{n}\right]}\right) \stackrel{*}{\rightarrow} \mu
$$

where $\mathcal{L}^{1}$ denotes the Lebesgue measure on $\mathbb{R}$. Further the convergence is understood to be the weak-* convergence in $C^{0}\left(T^{1} M, \mathbb{R}\right)^{\prime}$. By an elementary calculation we see that a limit measure is always $\Phi$-invariant.

Call a future pointing maximizer $\gamma: \mathbb{R} \rightarrow M$ a $\mathfrak{T}^{\circ}$-maximizer if it admits limit measures $\mu_{1}, \ldots, \mu_{b+1}$ with $\sum \rho\left(\mu_{i}\right) \in \mathfrak{T}^{\circ}$. Note that this definition is equivalent to the one given in [21]. It is not obvious at this point whether every $\mathfrak{T}^{\circ}$-maximizer admits a limit measure with rotation class in $\mathfrak{T}^{\circ}$. Following the Lorentzian Hedlund example in [21] this does not seem entirely impossible. 
Corollary 2.14. Let $(M, g)$ be of class $A_{1}$. Then any limit measure of a $\mathfrak{T}^{\circ}$ maximizer is supported entirely in $\operatorname{Time}(M, g)$.

For $\varepsilon>0$ set

$$
\operatorname{Time}(M, g)^{\varepsilon}:=\{v \in \operatorname{Time}(M, g)|\operatorname{dist}(v, \operatorname{Light}(M, g)) \geq \varepsilon| v \mid\} .
$$

The following result strengthens [21, Theorem 2.10, Corollary 2.22] in the class of class $A_{1}$ spacetimes. The proof will be given in Sect. 3.2.

Theorem 2.15. Let $(M, g)$ be of class $A_{1}$. Then there exist $\varepsilon>0$ and at least $b$ many maximal $\Phi$-ergodic probability measures $\mu_{1}, \ldots, \mu_{b}$ such that $\left\{\rho\left(\mu_{k}\right)\right\}_{1 \leq k \leq b}$ is a basis of $H_{1}(M, \mathbb{R})$ and

$$
\operatorname{supp} \mu_{k} \subseteq \operatorname{Time}(M, g)^{\varepsilon}
$$

for all $1 \leq k \leq b$.

The following corollary generalizes [18, Corollary 4.8] in a subclass of class $\mathrm{A}_{1}$ spacetimes. Note that globally conformally flat Lorentzian tori are trivially of class $\mathrm{A}_{1}$. The proof will be given in Sect. 3.2.

Corollary 2.16. Let $(M, g)$ be of class $A_{1}$ and assume that the stable time separation $\mathfrak{l}$ vanishes somewhere on $\partial \mathfrak{T} \backslash\{0\}$. Then there exist infinitely many maximal ergodic probability measures $\mu$ with

$$
\text { supp } \mu \in \operatorname{Time}(M, g) \text {. }
$$

\subsection{Lipschitz continuity of the time separation}

It is known, see [20, Theorem 16], that for class A spacetimes the time separation on the Abelian cover satisfies a coarse Lipschitz property. The above separation results for the support of certain maximal measures and therefore tangents to maximizers from the light cones have a direct implication for the geometry of the Abelian cover, improving the coarse Lipschitz property to a Lipschitz property. Note that by local considerations the Lipschitz property can never extend to the boundary of the set of pairs of endpoints of future pointing curves. The following result is to the authors knowledge the only result in this generality besides [6].

Theorem 2.17. Let $(M, g)$ be of class $A_{1}$. Then for all $\varepsilon>0$ there exist constants $K(\varepsilon), L(\varepsilon)<\infty$ such that the time separation

$$
(x, y) \mapsto d(x, y)
$$

of the Abelian cover $(\bar{M}, \bar{g})$ is $L(\varepsilon)$-Lipschitz on

$$
\left\{(x, y) \in \bar{M} \times \bar{M} \mid y-x \in \mathfrak{T}_{\varepsilon} \backslash B_{K(\varepsilon)}(0)\right\} .
$$


A few comments are in order on why the result is optimal for general class $A_{1}$ spacetimes. The flat torus is an example of a class $A_{1}$ spacetime for which the Lipschitz continuity of the time separation on the Abelian cover can not be extended to boundary of the causally related points.

Further the Lorentzian Hedlund examples in [21] show that the condition " $\| y-$ $x \| \geq K(\varepsilon)$ " is necessary for the Lipschitz continuity. Locally, i.e. for $\|y-x\|$ small, there exist $x, y \in \mathbb{R}^{3}$ with $d(x, y)=0$ and $y-x \in \mathfrak{T}_{\varepsilon}$ for some $\varepsilon>0$. At such a pair $(x, y)$ the time separation cannot be Lipschitz continuous.

\section{Proofs}

\subsection{Uniformly vicious Lorentzian manifolds}

Proof of Proposition 2.5. We consider the case of the manifold being diffeomorphic to $T^{2}$ only. The case of the Klein bottle follows from the torus case by a double covering. Then any uniform family for the lifted metric on $T^{2}$ gives rise to a uniform family on the Klein bottle via projection. Note that any finite cover of a vicious Lorentzian manifold is vicious again.

The other case follows similarly, since any vicious Lorentzian metric on $S^{1} \times \mathbb{R}$ gives rise to a partition of $S^{1} \times \mathbb{R}$ into essentially disjoint annuli with smooth timelike boundary curves. The uniform family can then be constructed on each annulus separately. If the construction is carried out carefully the local uniform families will join to a global uniform family.

Let $M \cong T^{2}$ and $g$ a vicious Lorentzian metric on $M$. We can assume w.l.o.g. that $(M, g)$ is time-oriented. Then [20, Proposition 4.6] implies that the stable time cone of $(M, g)$ has nonempty open interior.

Choose a pair of transversal future pointing timelike vector fields $X, Y$ on $M$ such that the rotation vectors of $X$ and $Y$ have different directions in the interior of the stable time cone. This can be easily achieved by choosing a future pointing timelike vector field $X$ and a nonsingular lightlike vector field $\bar{Y}$. The existence of $X$ follows from the time-orientability of $(M, g)$ and the existence of $Y$ follows from the time-orientability of $(M, g)$ and the orientability of $M$, see [19]. Since $M$ is compact, it is clear that the rotation vector of $X$ lies in the interior of the stable time cone. Then $Y:=X+\bar{Y}$ yields a future pointing timelike vector field with rotation vector different from $X$.

Note that every forward orbit of $X$ intersects every backward orbit of $Y$ infinitely many times, since the rotation vectors have different directions. Therefore by adjoining forward and backward orbits we can construct a continuous family of timelike loops covering $M$. Simply choose a fundamental class $\eta$ which is mapped into the interior of the cone over the roation vectors of $X$ and $Y$. Then there exists a positive multiple $\eta^{k}$ of $\eta$ such that for every $p \in M$ the intersection of the forward orbit of $X$ with the backward orbit of $Y$ through $p$ can be chosen such that the resulting timelike future pointing curve represents $\eta^{k}$. These curves depend continuously on $p$ since we have chosen $X$ and $Y$ to be transversal. Denote the constant arclength parameterization of the curve through $p$ on $S^{1}$ with $\gamma_{p}$. 
Now we can choose $\mathcal{M}=M$ and $H: M \times S^{1} \rightarrow M,(p, t) \mapsto \gamma_{p}(t)$. $H$ is clearly continuous, the loops $\gamma_{p}$ are timelike and $\left.H\right|_{M \times\{0\}}=\left.\mathrm{id}\right|_{M}$, i.e. a smooth surjective submersion.

Recall that we have fixed a complete Riemannian metric $g_{R}$ on $M$. Define for $p \in M$ the positive number $\operatorname{inj}(M, g)_{p}$ as the supremum over all $\eta>0$ such that $B_{\eta}(p)$ is contained in a convex normal neighborhood of $p$ in $(M, g)$ with $g_{R}$-diameter bounded from above by 1 . The diameter condition is posed solely for technical reasons. We can assume for the rest of the section that $(M, g)$ is time-oriented, see Sect. 2.2.

Lemma 3.1. Let $(M, g)$ be uniformly vicious. Then there exists a uniform family $H: \mathcal{M} \times S^{1} \rightarrow M$ smooth on a neighborhood of $\mathcal{M} \times\{0\}$ and such that the loops $\left.H\right|_{\{x\} \times S^{1}}$ are piecewise geodesic for all $x \in \mathcal{M}$.

Proof. Let $H_{0}: \mathcal{M} \times S^{1} \rightarrow M$ be a uniform family. Define

$$
i_{x}:=\inf _{t \in S^{1}}\left\{\operatorname{inj}(M, g)_{H_{0}(x, t)}\right\}
$$

for $x \in \mathcal{M}$. Note that $i_{x}$ is lower semicontinuous and positive for all $x \in \mathcal{M}$. Therefore we can choose a continuous function $\underline{i}: \mathcal{M} \rightarrow(0, \infty)$ with $\underline{i}(x) \leq i_{x}$ for all $x \in \mathcal{M}$. For $N \in \mathbb{N}$ set

$$
\begin{aligned}
& U_{N}:=\left\{x \in N \mid \operatorname{dist}\left(H_{0}(x, s), H_{0}(x, t)\right)<\left(2^{-1}-2^{-N}\right) \underline{i}(x),\right. \\
& \left.\forall s, t \in S^{1}:|s-t|<2^{-N}\right\} .
\end{aligned}
$$

$\left\{U_{N}\right\}_{N \in \mathbb{N}}$ is an open cover of $\mathcal{M}$ with $\overline{U_{N}} \subseteq U_{N+1}$. Choose $W_{N} \subseteq \mathcal{M}$ open with $\overline{U_{N}} \subseteq W_{N} \subseteq \overline{W_{N}} \subseteq U_{N+1}$ for every $N \in \mathbb{N}$. Then $\left\{U_{N+1} \backslash \overline{W_{N-1}}\right\}_{N \in \mathbb{N}}$ forms an open, locally finite cover of $\mathcal{M}$. Choose a partition of unity $\left\{\varphi_{N}\right\}_{N \in \mathbb{N}}$ subordinate to $\left\{U_{N+1} \backslash \overline{W_{N-1}}\right\}_{N \in \mathbb{N}}$. We use the following index convention

$$
\operatorname{supp} \varphi_{N} \subseteq U_{N+1} \backslash \overline{W_{N-1}} .
$$

Consider the set $\mathcal{B}$ of nonzero 0 -1-sequences $\alpha=a_{1} a_{2} \ldots$ which eventually become constant to 0 . Further consider the subset $\mathcal{B}_{N}$ of $\mathcal{B}$ whose elements are identically 0 beyond the $N$-th digit. Denote by $\beta_{N}$ the sequence which is identically 0 except for the $N$-th digit. Set $\left.r_{1} \equiv \mathrm{id}\right|_{\mathcal{B}_{1}}$ and for $N \geq 2$ define the following operations $r_{N}: \mathcal{B}_{N} \rightarrow \mathcal{B}_{N-1}$. For $\alpha=\beta_{N}$ set $r_{N}(\alpha)=\beta_{N-1}$ and for $\alpha \in$ $\mathcal{B}_{N} \backslash\left\{\beta_{N}\right\}$ define $r_{N}(\alpha)$ by setting the $N$-th digit to 0 .

For $\alpha \in \mathcal{B}$ choose $N \in \mathbb{N}$ with $\alpha \in \mathcal{B}_{N}$. Set $\alpha_{n}:=r_{n+1} \circ \cdots \circ r_{N}(\alpha)$ for $n \leq N-1$ and $\alpha_{n}:=\alpha$ for $n \geq N$. Note that this definition does not depend on the choice of $N$. Set $\bar{t}_{\alpha}:=\sum_{n=1}^{\infty} a_{n} 2^{-n}$, where $\alpha=a_{1} a_{2} \ldots$, and

$$
\bar{t}_{\alpha}(x):=\sum_{n=1}^{\infty} \bar{t}_{\alpha_{n}} \varphi_{n}(x) \in[0,1) .
$$

Note that $x \mapsto \bar{t}_{\alpha}(x)$ is smooth. The numbers $\bar{t}_{\alpha}(x)$ naturally define classes $t_{\alpha}(x) \in$ $S^{1}$. For the rest of the proof we will denote real numbers in $\mathbb{R}$ with $\bar{t}$ and with $t$ their projections to $S^{1} \cong \mathbb{R} / \mathbb{Z}$. 
Denote with $\omega_{N} \in \mathcal{B}$ the sequence which is identical to 1 for all digits smaller than or equal to $N$, and 0 everywhere else. Define the successor operation

$$
\alpha=a_{1} a_{2} \ldots \in \mathcal{B}_{N} \mapsto s_{N}(\alpha)=a_{1}^{\prime} a_{2}^{\prime} \ldots \in \mathcal{B}_{N}
$$

as follows. If $\alpha=\omega_{N}$ set $s_{N}(\alpha)=\omega_{N}$. If $a_{N}=0$ set $a_{i}^{\prime}=a_{i}$ for $i \leq N-1$ and $a_{N}^{\prime}=1$. If $\alpha \neq \omega_{N}$ and $a_{N}=1$ choose $1<k \leq N$ minimal such that $a_{i}=1$ for all $k \leq i \leq N$. In this case set $a_{i}^{\prime}=0$ for $k \leq i \leq N, a_{k-1}^{\prime}=1$ and $a_{j}^{\prime}=a_{j}$ for $j<k-1$.

Define the map $H_{N}: U_{N} \times S^{1} \rightarrow M$ as follows. Let $x \in U_{N}$. For $\alpha \in$ $\mathcal{B}_{N}$ define the curve $\gamma_{\alpha, x}:\left[\bar{t}_{\alpha}(x), \bar{t}_{S_{N}(\alpha)}(x)\right] \rightarrow M$ to be the unique geodesic connecting $H_{0}\left(x, t_{\alpha}(x)\right)$ with $H_{0}\left(x, t_{s_{N}(\alpha)}(x)\right)$. Note that $\bar{t}_{\alpha}(x) \leq \bar{t}_{s_{N}(\alpha)}(x)$ and $\left|t_{\alpha}(x)-t_{s_{N}(\alpha)}(x)\right| \leq 2^{-n}$ for $x \in U_{n}$. Therefore

$$
\operatorname{dist}\left(H_{0}\left(x, t_{\alpha}(x)\right), H_{0}\left(x, t_{s_{N}(\alpha)}(x)\right)\right) \leq i_{x} / 2
$$

and $\gamma_{\alpha, x}$ is well defined. By Definition 2.3, the geodesic $\gamma_{\alpha, x}$ is timelike if and only if $\gamma_{x, \alpha}$ is non-constant if and only if $\bar{t}_{s_{N}(\alpha)}(x)>\bar{t}_{\alpha}(x)$.

Define $\bar{t}_{\omega}, \bar{t}_{\beta}: \mathcal{M} \rightarrow \mathbb{R}$ by setting $\left.\bar{t}_{\beta}\right|_{U_{N-1}}:=\left.\bar{t}_{\beta_{N}}\right|_{U_{N-1}}$ and $\left.\bar{t}_{\omega}\right|_{U_{N-1}}:=$ $\left.\bar{t}_{\omega_{N}}\right|_{U_{N-1}} \cdot \bar{t}_{\beta}$ and $\bar{t}_{\omega}$ are well defined smooth functions, since $\left.\bar{t}_{\beta_{N+1}, \omega_{N+1}}\right|_{U_{N-1}} \equiv$ $\left.\bar{t}_{\beta_{N}, \omega_{N}}\right|_{U_{N-1}}$, and induce smooth functions $t_{\beta}, t_{\omega}: \mathcal{M} \rightarrow S^{1}$. Now define (note that $\left.r_{N}\left(\omega_{N}\right)=\omega_{N-1}\right)$

$H_{N}: U_{N} \times S^{1} \rightarrow M, \quad H_{N}(x, t):= \begin{cases}H_{0}(x, t) & \text { if } t \in\left[t_{\omega}(x), t_{\beta}(x)\right] \text { and } \\ \gamma_{\alpha, x}(t) & \text { if } t \in\left[t_{\alpha}(x), t_{S_{N}}(\alpha)\right. \\ & (x)] \text { for } \alpha \in \mathcal{B}_{N} .\end{cases}$

$H_{N}$ is continuous by construction and we have $\left.\left.H_{N+1}\right|_{U_{N-1}} \equiv H_{N}\right|_{U_{N-1}}$. Therefore we can define a map $H^{\prime}: \mathcal{M} \times S^{1} \rightarrow M$ with $\left.\left.H^{\prime}\right|_{U_{N-1}} \equiv H_{N}\right|_{U_{N-1}}$. $H^{\prime}$ satisfies the claim of the lemma on $\left(\mathcal{M} \times S^{1}\right) \backslash U$, where

$$
U:=\left\{(x, t) \in \mathcal{M} \times S^{1} \mid t \in\left(t_{\omega}(x), t_{\beta}(x)\right)\right\} .
$$

The reason why we have not altered $\left.H_{0}\right|_{U}$ so far, is that we want to retain the property that $\left.H_{0}\right|_{\mathcal{M} \times\{0\}}$ is a surjective submersion. In order to do so we have to be more careful with our construction on $U$. Choose for every $x \in \mathcal{M}$ a geodesically convex normal neighborhood $V_{x}$ of $H_{0}(x, 0)$ with $\operatorname{diam}_{g_{R}}\left(V_{x}\right) \leq 1$ such that $B_{i_{x}}\left(H_{0}(x, 0)\right) \subseteq V_{x}$. Next choose a smooth map $\chi: \mathcal{M} \times\{0\} \rightarrow$ Time $(M, g)$, see Sect. 2.2, over $\left.H_{0}\right|_{\mathcal{M} \times\{0\}}$, i.e. $\chi(x, 0) \in \operatorname{Time}(M, g)_{H_{0}(x, 0)}$ for all $x \in \mathcal{M}$. Consider the (future pointing timelike) geodesic $c_{x}$ starting in $H_{0}(x, 0)$ with direction $\chi(x, 0)$ and for $N \in \mathbb{N}$ the set

$$
V_{N}:=\left\{x \in \mathcal{M} \mid c_{x}\left( \pm N^{-1}\right) \in I_{V_{x}}^{\mp}\left(H_{0}\left(x, t_{\beta, \omega}(x)\right)\right) \text { and } N^{-1}<\left|\bar{t}_{\beta, \omega}(x)\right|\right\} .
$$

$\left\{V_{N}\right\}_{N \in \mathbb{N}}$ is an open cover of $\mathcal{M}$ and we have $\overline{V_{N}} \subseteq V_{N+1}$. Choose open sets $Z_{N}$ with $\overline{V_{N}} \subseteq Z_{N} \subseteq \overline{Z_{N}} \subseteq V_{N+1}$. Then $\left\{V_{N+1} \backslash \overline{Z_{N-1}}\right\}_{N \in \mathbb{N}}$ forms an open, locally finite covering of $\mathcal{M}$. Choose a partition of unity $\left\{\psi_{N}\right\}_{N \in \mathbb{N}}$ subordinate to this covering. We again use the index convention

$$
\operatorname{supp} \psi_{N} \subseteq V_{N+1} \backslash \overline{Z_{N-1}} \text {. }
$$


Set $\bar{\tau}_{x}:=\sum_{N=1}^{\infty}(N+1)^{-1} \psi_{N}(x) \in(0,1)$ and denote with $\tau_{x}$ the natural projection to $S^{1}$. For $x \in \mathcal{M}$ denote with

$$
\zeta_{x}^{+}:\left[\bar{\tau}_{x}, \bar{t}_{\beta}(x)\right] \rightarrow M \text { and } \zeta_{x}^{-}:\left[\bar{t}_{\omega}(x), 1-\bar{\tau}_{x}\right] \rightarrow M
$$

the unique geodesics connecting $c_{x}\left(\bar{\tau}_{x}\right)$ with $H_{0}\left(x, t_{\beta}(x)\right)$ and $H_{0}\left(x, t_{\omega}(x)\right)$ with $c_{x}\left(-\bar{\tau}_{x}\right)$, respectively. Note that $\zeta_{x}^{ \pm}$are futurepointing timelike for all $x \in \mathcal{M}$. Define

$$
H(x, t):= \begin{cases}H^{\prime}(x, t) & \text { if } t \in\left[t_{\beta}(x), t_{\omega}(x)\right] \\ \zeta_{x}^{+}(\bar{t}) & \text { if } \bar{t} \in\left[\bar{\tau}_{x}, \bar{t}_{\beta}(x)\right] \\ \zeta_{x}^{-}(\bar{t}) & \text { if } \bar{t} \in\left[\bar{t}_{\omega}(x), 1-\bar{\tau}_{x}\right] \text { and } \\ c_{x}(\bar{t}) & \text { if } \bar{t} \in\left[0, \bar{\tau}_{x}\right] \cup\left[1-\bar{\tau}_{x}, 1\right] .\end{cases}
$$

$\left.H\right|_{\{x\} \times S^{1}}$ is a piecewise geodesic, essentially timelike loop for all $x \in \mathcal{M}$. Since $H$ coincides with $H_{0}$ on $\mathcal{M} \times\{0\}$, we know that $\left.H^{\prime \prime}\right|_{\mathcal{M} \times\{0\}}$ is a surjective submersion.

By construction we have $\sup _{(x, t)} \operatorname{dist}\left(H_{0}(x, t), H(x, t)\right) \leq 1$. Recall that all convex normal neighborhoods were assumed to have $g_{R}$-diameter bounded by 1 . Therefore we have

$$
H^{-1}(K) \subseteq H^{-1}\left(B_{1}(K)\right)
$$

for every compact set $K \subseteq M$. Consequently $H$ is a proper map as well.

Definition 3.2. Let $(M, g)$ be a spacetime and $\varepsilon>0$.

(i) A future pointing curve $\gamma: I \rightarrow M$ is $\varepsilon$-timelike if

$$
\dot{\gamma}(t) \in \operatorname{Time}(M, g)_{\gamma(t)}^{\varepsilon}
$$

for almost all $t \in I$.

(ii) A past pointing curve is said to be $\varepsilon$-timelike if it is $\varepsilon$-timelike for the reversed parametrization.

The definition of $\varepsilon$-timelikeness is independent of the Riemannian metric in the following sense: Let $g_{R}, \widetilde{g}_{R}$ be equivalent Riemannian metrics on $M$, i.e. there exist $0<c \leq C<\infty$ such that $c \widetilde{g}_{R} \leq g_{R} \leq C \widetilde{g}_{R}$. Then every, relative to $\widetilde{g}_{R}$, $\widetilde{\varepsilon}$-timelike curve is $\frac{c}{C} \widetilde{\varepsilon}$-timelike relative to $g_{R}$.

Proposition 3.3. Let $K \subseteq M$ be compact and let $\gamma: I \rightarrow K$ be a future pointing curve. Then $\gamma$ is $\varepsilon$-timelike for some $\varepsilon>0$ if and only if $\exp _{\gamma(s)}^{-1}(\gamma(t)) \in$ Time $(M, g)^{\delta}$ for some $\delta>0$ and all $s<t \in I$ sufficiently close.

For the proof we will need the following elementary estimates: There exist constants $0<\widetilde{c}<\widetilde{C}<\infty$, depending only on $g$ and $g_{R}$ and $K$, such that

$$
\widetilde{c}|v| \operatorname{dist}\left(v, \operatorname{Light}(M, g)_{p}\right) \leq\left|g_{p}(v, v)\right| \leq \widetilde{C}|v| \operatorname{dist}\left(v, \operatorname{Light}(M, g)_{p}\right)
$$

for all $p \in K$ and all future pointing $v \in T M_{p}$. The proof is elementary and can be found in [18]. 
Proof. If we assume $\exp _{\gamma(s)}^{-1}(\gamma(t)) \in \operatorname{Time}(M, g)^{\delta}$ for some $\delta>0$ and all $s<t \in$ $I$ sufficiently close, we obtain $\dot{\gamma}(s) \in \operatorname{Time}(M, g)^{\delta}$ for almost all $t$. Therefore $\gamma$ is $\delta$-timelike.

Conversely assume that $\gamma$ is $\varepsilon$-timelike for some $\varepsilon>0$. Then we have $L^{g}(\gamma) \geq$ $\varepsilon_{1} L^{g_{R}}(\gamma)$ for some $\varepsilon_{1}>0$. This follows from (1) for $v=\dot{\gamma}$ and $\varepsilon_{1}:=\sqrt{\widetilde{c} \varepsilon}$.

Now consider $s<t \in I$ such that $\left.\gamma\right|_{[s, t]}$ is contained in a compact, convex normal neighborhood $U_{s}$ of $\gamma(s)$ such that $\left(\bar{U}_{s},\left.g\right|_{U_{s}}\right)$ is globally hyperbolic and $\exp _{\gamma(s)}^{-1}$ is bi-Lipschitz on $U_{s}$. Then we have

$$
d_{U_{s}}(\gamma(s), \gamma(t)) \geq L^{g}\left(\left.\gamma\right|_{[s, t]}\right) \geq \varepsilon_{1} L^{g_{R}}\left(\left.\gamma\right|_{[s, t]}\right) \geq \varepsilon_{1} \operatorname{dist}(\gamma(s), \gamma(t)) .
$$

Since $\exp _{\gamma(s)}^{-1}$ is bi-Lipschitz on $U_{s}$, we have

$$
\operatorname{dist}(\gamma(s), \gamma(t)) \geq \varepsilon_{2}\left|\exp _{\gamma(s)}^{-1}(\gamma(t))\right|
$$

for some $\varepsilon_{2}>0$ and therefore

$$
\begin{aligned}
d^{2}(\gamma(s), \gamma(t)) & =-g\left(\exp _{\gamma(s)}^{-1}(\gamma(t)), \exp _{\gamma(s)}^{-1}(\gamma(t))\right) \\
& \leq \widetilde{C}\left|\exp _{\gamma(s)}^{-1}(\gamma(t))\right| \operatorname{dist}\left(\exp _{\gamma(s)}^{-1}(\gamma(t)), \operatorname{Light}(M, g)\right)
\end{aligned}
$$

The claim now follows for $\delta:=\frac{\varepsilon_{1}^{2} \varepsilon_{2}^{2}}{\widetilde{C}}$.

Lemma 3.4. Let $V \subseteq \mathbb{R}^{m}$ be open, $M$ a submanifold of $V$ and $g$ a time-oriented Lorentzian metric on $V$ such that the restriction of $g$ to $M$ is Lorentzian as well. Further let $\varepsilon>0, \mathcal{N}$ be a smooth n-manifold and $H: \mathcal{N} \times S^{1} \rightarrow V$ a continuous map such that $\left.H\right|_{\{y\} \times S^{1}}$ is an $\varepsilon$-timelike loop for all $y \in \mathcal{N}, H$ is smooth on a neighborhood of $\mathcal{N} \times\{0\}$ and the map $\left.H\right|_{\mathcal{N} \times\{0\}}$ is a submersion. Then for every $x \in \mathcal{N}$ there exists a neighborhood $U_{x}$ of $x$ such that for all $\delta \in(0, \varepsilon)$ there exists a smooth map $\widetilde{H}_{x, \delta}: U_{x} \times S^{1} \rightarrow V$ with $\left.\widetilde{H}_{x, \delta}\right|_{\{y\} \times S^{1}}$ is $(\varepsilon-\delta)$-timelike for all $y \in U_{x}$, $\left|\widetilde{H}_{x, \delta}(y, t)-H(y, t)\right| \leq \delta$ for all $(y, t) \in U_{x} \times S^{1}$ and $|d \widetilde{H}(y, 0)-d H(y, 0)| \leq \delta$ for all $y \in U_{x}$.

We will denote with $B_{r}^{n} \subseteq \mathbb{R}^{n}$ the open ball of radius $r>0$ and center $0 \in \mathbb{R}^{n}$.

Proof. The statement is local, therefore we can assume $\mathcal{N} \cong \mathbb{R}^{n}$. Throughout the proof we will identify the tangent spaces $T V_{p}$ with $\mathbb{R}^{m}$. Both $\mathbb{R}^{n}$ and $V$ are equipped with the standard scalar product as Riemannian metric. For $p \in V$ we will denote with Time $\left(\mathbb{R}^{m}, g_{p}\right)$ the future pointing timelike vectors in $\left(\mathbb{R}^{m}, g_{p}\right) \cong\left(T V_{p}, g_{p}\right)$. Time $\left(\mathbb{R}^{m}, g_{p}\right)^{\varepsilon}$ is defined in the obvious way. W.l.o.g. we can assume that the loops $\left.H\right|_{\{x\} \times S^{1}}$ are future pointing for all $x \in \mathcal{N}$. Note that by assumption $\mathcal{N}$ is connected.

For given $x \in \mathbb{R}^{n}$ and $\delta>0$ choose a real number $0<\eta<\delta$ and a compact neighborhood $K \subseteq \mathbb{R}^{n}$ of $x$ such that

$$
H(z, t)-H(z, s) \in \operatorname{Time}\left(\mathbb{R}^{m}, g_{p}\right)^{\varepsilon-\delta} \cup\{0\},
$$

for all $z \in K, s, t \in S^{1}$ and $p \in V$ such that $|p-H(z, s)|,|s-t| \leq \eta$ and $\left.H\right|_{\{z\} \times[s, t]}$ is future pointing. Note that under these assumptions $H(z, t)-H(z, s)=0$ if and 
only if $s=t$, since $\left.H\right|_{\{z\} \times S^{1}}$ is causal. This choice is possible since the loops $\left.H\right|_{\{z\} \times S^{1}}$ are $\varepsilon$-timelike and we can apply the Fundamental Theorem of Calculus to any arclength parameterization of $\left.H\right|_{\{z\} \times S^{1}}$.

Choose $\frac{1}{2}>\kappa^{\prime}>0$ such that $|H(y, s)-H(z, t)|<\frac{\eta}{2}$ for all $(y, s),(z, t) \in$ $K \times S^{1}$ with $|y-z|,|t-s|<\kappa^{\prime}$. Further choose smooth functions $\varphi: \mathbb{R}^{n} \rightarrow[0, \infty)$ and $\tilde{\theta}: \mathbb{R} \rightarrow[0, \infty)$ with $\operatorname{supp} \varphi \subseteq B_{1}^{n}$, supp $\widetilde{\theta} \subseteq B_{1}^{1}$ and $\int_{\mathbb{R}^{n}} \varphi=\int_{\mathbb{R}} \tilde{\theta}=1$. For $0<\kappa<\kappa^{\prime} \operatorname{set} \varphi^{\kappa}(x):=\kappa^{-n} \varphi\left(\kappa^{-1} x\right)$ and $\widetilde{\theta}^{\kappa}(t):=\kappa^{-1} \widetilde{\theta}\left(\kappa^{-1} t\right)$. Define functions $\bar{\varphi}^{\kappa}: \mathbb{R}^{n} \times \mathbb{R}^{n} \rightarrow[0, \infty),\left(y^{\prime}, y\right) \mapsto \varphi^{\kappa}\left(y^{\prime}-y\right)$ and $\bar{\theta}^{\kappa}: \mathbb{R} \times \mathbb{R} \rightarrow[0, \infty)$, $\left(t^{\prime}, t\right) \mapsto \widetilde{\theta}^{\kappa}\left(t^{\prime}-t\right)$. Since we have $\operatorname{supp} \widetilde{\theta}^{\kappa} \subseteq\left(-\frac{1}{2}, \frac{1}{2}\right)$ (recall $\left.\kappa<\frac{1}{2}\right)$, the function $\theta^{\kappa}: S^{1} \times S^{1} \rightarrow[0, \infty),\left(t^{\prime}, t\right) \mapsto \bar{\theta}^{\kappa}\left(\bar{t}^{\prime}-\bar{t}\right)$ is well defined, where $\bar{t}, \bar{t}^{\prime} \in \mathbb{R}$ are lifts of $t$ and $t^{\prime}$, resp., with $\left|\bar{t}^{\prime}-\bar{t}\right|<1$. Define for $0<\kappa<\kappa^{\prime}$

$$
\widetilde{H}_{x, \kappa}: K \times S^{1} \rightarrow V,(y, t) \mapsto \int_{\mathbb{R}^{n} \times S^{1}} H\left(y^{\prime}, t^{\prime}\right) \bar{\varphi}^{\kappa}\left(y^{\prime}, y\right) \theta^{\kappa}\left(t^{\prime}, t\right) d y^{\prime} d t^{\prime} .
$$

Our goal is to show that the loops $\left.\widetilde{H}_{x, \kappa}\right|_{\{y\} \times S^{1}}$ are $(\varepsilon-\delta)$-timelike loops for all $y \in K$ and $\kappa$ sufficiently small. We have

$$
\left|\widetilde{H}_{x, \kappa}(y, t)-H(y, t)\right| \leq \int_{\mathbb{R}^{n} \times S^{1}} \bar{\varphi}^{\kappa}\left(y^{\prime}, y\right) \theta^{\kappa}\left(t^{\prime}, t\right)\left|H\left(y^{\prime}, t^{\prime}\right)-H(y, t)\right| d y^{\prime} d t^{\prime} \leq \frac{\eta}{2}
$$

for all $(y, t) \in K \times S^{1}$ by our assumption above. Recall that, by definition of $\theta$, we have $\theta^{\kappa}(t+\tau, t)=\widetilde{\theta}^{\kappa}(\bar{\tau})$ for all $t, \tau \in S^{1}$ and $\kappa<\kappa^{\prime}$, where $\bar{\tau}$ is the unique lift of $\tau$ to $\left(-\frac{1}{2}, \frac{1}{2}\right]$. Then we have

$$
\begin{aligned}
& \widetilde{H}_{x, \kappa}(y, t)-\widetilde{H}_{x, \kappa}(y, s) \\
& \quad=\int_{\mathbb{R}^{n}} \bar{\varphi}^{\kappa}\left(y^{\prime}, y\right)\left[\int_{S^{1}} H\left(y^{\prime}, t^{\prime}\right) \theta^{\kappa}\left(t^{\prime}, t\right) d t^{\prime}-\int_{S^{1}} H\left(y^{\prime}, s^{\prime}\right) \theta^{\kappa}\left(s^{\prime}, s\right) d s^{\prime}\right] d y^{\prime} \\
& \quad=\int_{\mathbb{R}^{n}} \bar{\varphi}^{\kappa}(z, y) \int_{S^{1}}[H(z, t+\tau)-H(z, s+\tau)] \widetilde{\theta}^{\kappa}(\bar{\tau}) d \tau d z
\end{aligned}
$$

for all $s, t \in S^{1}$ and $y \in K$.

Recall that we have $|H(z, s+\tau)-H(y, s)|<\eta / 2$ if $|z-y|$ and $|\tau|<\kappa$. Consequently we have $H(z, s+\tau) \in B_{\eta}\left(\widetilde{H}_{x, \kappa}(y, s)\right)$ and we get

$$
H(z, t+\tau)-H(z, s+\tau) \in \operatorname{Time}\left(\mathbb{R}^{m}, g_{\widetilde{H}_{x, \kappa}(y, s)}\right)^{\varepsilon-\delta} \cup\{0\}
$$

by (2), for all $z \in K$ and $t$ such that $|s-t| \leq \eta$ and $\left.H\right|_{\{z\} \times[s+\tau, t+\tau]}$ is future pointing. Using (3) and the fact that $\operatorname{Time}\left(\mathbb{R}^{m}, g_{\widetilde{H}_{x, \kappa}(y, s)}\right)^{\varepsilon-\delta}$ is a convex cone, we obtain

$$
\widetilde{H}_{x, \kappa}(y, t)-\widetilde{H}_{x, \kappa}(y, s) \in \operatorname{Time}\left(\mathbb{R}^{m}, g_{\widetilde{H}_{x, \kappa}(y, s)}\right)^{\varepsilon-\delta} \cup\{0\} .
$$

Since $\widetilde{H}_{x, \kappa}$ is smooth and Time $(V, g)_{\tilde{H}_{x, \kappa}(y, s)}^{\varepsilon-\delta}$ is closed, we get

$$
\partial_{t} \widetilde{H}_{x, \kappa}(y, s) \in \operatorname{Time}(V, g)^{\varepsilon-\delta} \cup\{0\}
$$


for all $(y, s) \in K \times S^{1}$. It is now easy to see that $\partial_{t} \widetilde{H}_{x, \delta}$ is timelike on a sufficiently small neighborhood $U_{x}$ of $x$.

The only thing left to note is that this approximation procedure applies to any $C^{r}$-topology, i.e. the differentials of $H$ at points $(y, t)$ are approximated by the differentials of $\widetilde{H}$ as well. This completes the proof.

At this point we fix a complete Riemannian metric $\mathcal{G}_{R}$ on $\mathcal{M}$ once and for all. The following proof is closely oriented on the smoothing technique presented in [9].

Proof of Proposition 2.6. Let $H_{0}: \mathcal{M} \times S^{1} \rightarrow M$ be a uniform family. We will reduce the claim to the case that $M$ is a submanifold of some $\mathbb{R}^{m^{\prime}}$. For this choose an embedding $F: M \rightarrow \mathbb{R}^{m^{\prime}}$ for some $m^{\prime} \geq 2 m$. Consider $\mathbb{R}^{m^{\prime}}$ to be equipped with the standard Riemannian metric $\langle.,$.$\rangle . Further consider the normal bundle$ $\pi_{N}: N \rightarrow F(M)$ of $F(M)$ and the exponential map $\exp ^{\perp}$ restricted to $N$. i.e. $\exp ^{\perp}: N \rightarrow \mathbb{R}^{m^{\prime}}, v \mapsto \pi_{N}(v)+v$. Choose a smooth function $\varepsilon: M \rightarrow(0, \infty)$ and a neighborhood $V_{N}$ of the zero section in $N$ such that $\left.\exp ^{\perp}\right|_{V_{N}}: V_{N} \rightarrow$ $\cup_{p \in M} B_{\varepsilon(p)}(F(p))=: V$ is a diffeomorphism. Next define on $V$ the Lorentzian metric

$$
g^{\prime}:=\left(\exp ^{\perp}\right)_{*}\left(F_{*} g+\left.\langle., .\rangle\right|_{N}\right) .
$$

Time-orient $g^{\prime}$ such that the embedding $F:(M, g) \rightarrow\left(V, g^{\prime}\right)$ preserves timeorientation. Note that, by definition, we have

$$
\left(\pi_{N} \circ\left(\exp ^{\perp}\right)^{-1}\right)_{*}: \operatorname{Time}\left(V, g^{\prime}\right) \rightarrow \operatorname{Time}\left(F(M), F_{*} g\right),
$$

and therefore timelike curves in $\left(V, g^{\prime}\right)$ are mapped to timelike curves by $\pi_{N} \circ$ $\left(\exp ^{\perp}\right)^{-1}$. Furthermore note that $\pi_{N}$ is 1-Lipschitz relative to the Riemannian metrics $g_{R}^{\prime}:=\left(\exp ^{\perp}\right)_{*}\left(F_{*} g_{R}+\left.\langle.,\rangle\right|_{N}.\right)$ and $g_{R}$. Consequently any smooth map $\bar{H}: \mathcal{M} \times S^{1} \rightarrow V$, such that the loops $\left.\bar{H}\right|_{\{x\} \times S^{1}}$ are timelike, projects to a smooth map $\bar{H}^{\prime}: \mathcal{M} \times S^{1} \rightarrow F(M)$ such that the loops $\left.H^{\prime}\right|_{\{x\} \times S^{1}}$ are timelike. We can further choose $\bar{H}$ such that $H^{\prime \prime}$ is still a surjective submersion onto $F(M)$. Thus $H^{\prime \prime}$ is a smooth uniform family on $F(M)$ and consequently induces such a family on $M$.

Choose bounded open neighborhoods $Z_{x}, W_{x}$ and $U_{x}$ of $x$ such that $\bar{Z}_{x} \subseteq W_{x} \subseteq$ $\bar{W}_{x} \subseteq U_{x}$ and Lemma 3.4 applies to $\left.H\right|_{U_{x} \times S^{1}}$. Choose a locally finite subcovering $\left\{Z_{i}\right\}_{i \in \mathbb{N}}\left(Z_{i}:=Z_{x_{i}}, W_{i}:=W_{x_{i}}, U_{i}:=U_{x_{i}}\right)$ of $\mathcal{M}$. We define inductively smooth maps $\bar{H}_{j}: \mathcal{M} \times S^{1} \rightarrow V$ with

(1) ${ }_{j} \bar{H}_{j} \equiv \bar{H}_{j-1}$ on $\left(\mathcal{M} \backslash W_{j}\right) \times S^{1}$.

(2) ${ }_{j} \bar{H}_{j}$ is smooth on $\cup_{i=1}^{j} Z_{i} \times S^{1}$ and $\left.\bar{H}_{j}\right|_{\{x\} \times S^{1}}$ is a smooth timelike loop for all $x \in \cup_{i=1}^{j} Z_{i}$.

(3) ${ }_{j} \bar{H}_{j}$ is smooth on a neighborhood of $\mathcal{M} \times\{0\}$ and the projection of $\bar{H}_{j} \mid \mathcal{M} \times\{0\}$ to $F(M)$ is a surjective submersion onto $F(M)$.

Since $\left\{Z_{i}\right\}$ is a locally finite cover, the sequence $\left\{\bar{H}_{j}\right\}$ converges on compact subsets of $\mathcal{M} \times S^{1}$ to a smooth map $\bar{H}: \mathcal{M} \times S^{1} \rightarrow V$ such that the loops $\left.\bar{H}\right|_{\{x\} \times S^{1}}$ are 
timelike. Therefore the only thing left to prove is the existence of a sequence $\left\{\bar{H}_{j}\right\}$ satisfying $(1)_{j}-(3)_{j}$ for all $j$.

For $j=0$ we have $\bar{H}_{0} \equiv H$ and there is nothing to prove. By Lemma 3.1 we can assume that every loop $\left.H_{0}\right|_{\{y\} \times S^{1}}$ is piecewise geodesic. Suppose now that $j>0$ and we have smooth maps $\bar{H}_{i}$ satisfying $(1)_{i}-(3)_{i}$ for $0 \leq i<j$. We can choose $\varepsilon_{j-1}>0$ such that every loop $\left.\bar{H}_{j-1}\right|_{\{y\} \times S^{1}}$ is $\varepsilon_{j-1}$-timelike for $y \in W_{j}$. Consider for $e_{j-1}>\delta>0$ approximations $\widetilde{H}_{j, \delta}: W_{j} \times S^{1} \rightarrow V$ of $\bar{H}_{j-1}$ according to Lemma 3.4.

We know that the loops $\left.\widetilde{H}_{j, \delta}\right|_{\{y\} \times S^{1}}$ are $\left(\varepsilon_{j-1}-\delta\right)$-timelike for all $y \in W_{j}$. Consequently we can choose $\delta_{j}>0$ such that $v+\left.\bar{H}_{j-1}\right|_{\{y\} \times S^{1}}$ and $v+\left.\widetilde{H}_{j, \delta}\right|_{\{y\} \times S^{1}}$ is $\varepsilon_{j-1} / 2$-timelike for all $y \in W_{j}$ and all $v \in B_{\delta_{j}}^{m^{\prime}}$. Choose a partition of unity $\left\{\lambda_{1}, \lambda_{2}\right\}$ subordinate to $\left\{W_{j}, \mathcal{M} \backslash \bar{Z}_{j}\right\}$. Define

$$
\bar{H}_{j}(y, t):=\lambda_{1}(y) \widetilde{H}_{j, \delta_{j}}(y, t)+\lambda_{2}(y) \bar{H}_{j-1}(y, t) .
$$

We have $\left.\left.\bar{H}_{j}\right|_{\left[\mathcal{M} \backslash W_{j}\right] \times S^{1}} \equiv \bar{H}_{j-1}\right|_{\left[\mathcal{M} \backslash W_{j}\right] \times S^{1}},\left.\bar{H}_{j}\right|_{\left[\cup_{i=1}^{j} Z_{i}\right] \times S^{1}}$ is smooth and $\left.\bar{H}_{j}\right|_{\{x\} \times S^{1}}$ is a smooth timelike loop for all $x \in \cup_{i=1}^{j} Z_{i} \times S^{1}$. Therefore $\bar{H}_{j}$ satisfies $(1)_{j}$ and (2) $)_{j}$. By the assumptions on $\bar{H}_{j-1}$ we know that $\bar{H}_{j}$ is smooth in a neighborhood of $\mathcal{M} \times\{0\}$. For $\delta_{j}$ sufficiently small, we know that the projection of $\bar{H}_{j}$ to $F(M)$ is a surjective submersion. This is a consequence of the standard approximation arguments in [9].

This completes the induction and the proof.

\subsection{Class $A_{1}$ spacetimes}

Recall from Sect. 3.1 that we have chosen a Riemannian metric $\mathcal{G}_{R}$ on $\mathcal{M}$. Note that by the compactness assumption the metric is always complete. $\mathcal{G}_{R}$ naturally induces a Riemannian metric on $T \mathcal{M}$ such that the canonical projection $\pi_{T \mathcal{M}}: T \mathcal{M} \rightarrow \mathcal{M}$ is a Riemannian submersion. Denote with $\mathcal{Z}$ the zero section of $T \mathcal{M}$. We define a bundle map

$$
X_{H}:(T \mathcal{M} \backslash \mathcal{Z}) \times S^{1} \rightarrow T\left(\mathcal{M} \times S^{1}\right)
$$

over the identity on $\mathcal{M} \times S^{1}$ as follows:

Consider for $(v, \varphi) \in(T \mathcal{M} \backslash \mathcal{Z}) \times S^{1}$ the quadratic form

$$
b_{(v, \varphi)}: \mathbb{R}^{2} \rightarrow \mathbb{R},(\lambda, \eta) \mapsto H^{*} g\left(\lambda v+\eta \partial_{\varphi}, \lambda v+\eta \partial_{\varphi}\right) .
$$

The equation $b_{(v, \varphi)}(\lambda, \eta)=0$ admits nontrivial solutions for all $(v, \varphi) \in$ $(T \mathcal{M} \backslash \mathcal{Z}) \times S^{1}$, since $b_{(v, \varphi)}$ is either indefinite (if and only if $r k\left(\left.H_{*}\right|_{\operatorname{span}\left\{v, \partial_{\varphi}\right\}}\right)=2$ ) or negative semidefinite (if and only if $r k\left(\left.H_{*}\right|_{\operatorname{span}\left\{v, \partial_{\varphi}\right\}}\right)=1$ ), but not negative definite. Note that $r k\left(\left.H_{*}\right|_{\operatorname{span}\left\{v, \partial_{\varphi}\right\}}\right) \geq 1$, since $H_{*}\left(\partial_{\varphi}\right)$ is always timelike and therefore $g\left(H_{*}\left(\partial_{\varphi}\right), H_{*}\left(\partial_{\varphi}\right)\right)<0$. Thus we have $b_{(v, \varphi)}(0, \eta)<0$ for all $(v, \varphi)$ and $\eta \neq 0$. In the case that $r k\left(\left.H_{*}\right|_{\operatorname{span}\left\{v, \partial_{\varphi}\right\}}\right)=2$, the set of solutions consists of two transversal one-dimensional subspaces which depend locally Lipschitz on $(v, \varphi)$. 
For every $(v, \varphi) \in(T \mathcal{M} \backslash \mathcal{Z}) \times S^{1}$ we define $X_{H}(v, \varphi):=v+\eta \partial_{\varphi}$ as the unique vector such that $\eta$ is maximal among all solutions $(1, \eta)$ of $b_{(v, \varphi)}(1, \eta)=0$. Then $H_{*}\left(X_{H}\right)$ is future pointing, if $r k\left(\left.H_{*}\right|_{\operatorname{span}\left\{v, \partial_{\varphi}\right\}}\right)=2$ and vanishes, if $r k\left(\left.H_{*}\right|_{\operatorname{span}\left\{v, \partial_{\varphi}\right\}}\right)=1$. The map $X_{H}$ is well defined and continuous for all $(v, \varphi) \in(T \mathcal{M} \backslash \mathcal{Z}) \times S^{1}$. Note that $X_{H}$ is locally Lipschitz on the set $\{(v, \varphi) \in$ $\left.(T \mathcal{M} \backslash \mathcal{Z}) \times S^{1} \mid r k\left(\left.H_{*}\right|_{\operatorname{span}\left\{v, \partial_{\varphi}\right\}}\right)=2\right\}$. Since $\mathcal{M}$ is compact, we can choose $L<\infty$ and $\varepsilon>0$ such that $X_{H}$ is $L$-Lipschitz on the $\varepsilon$-neighborhood of $H_{*}^{-1}\left(\operatorname{Light}(M, g) \cap T^{1} M\right) \cap\left(\operatorname{ker} H_{*}\right)^{\perp}$. This is due to the fact that $H_{*}\left(\partial_{\varphi}\right)$ is timelike.

Denote with $h_{H} \in \mathfrak{T}^{\circ}$ the homology class of the curves $\left.H\right|_{\{p\} \times S^{1}}$. The fact that $h_{H} \in \mathfrak{T}^{\circ}$ follows with a simple pertubation argument.

Proof of Theorem 2.11. Let $h \in \partial \mathfrak{T} \backslash\{0\}$ and $\left\{\lambda_{n}\right\}_{n \in \mathbb{N}}$ be a sequence of positive real numbers diverging to $\infty$. According to [20, Proposition 8] there exists a constant $\operatorname{err}\left(g, g_{R}\right)<\infty$ such that we can choose a sequence of future pointing lightlike maximizers $\gamma_{n}:\left[-T_{n}, T_{n}\right] \rightarrow M$ with $\left|\gamma_{n}^{\prime}\right| \equiv 1$ and

$$
\left\|\gamma_{n}\left(T_{n}\right)-\gamma_{n}\left(-T_{n}\right)-\lambda_{n} h\right\| \leq \operatorname{err}\left(g, g_{R}\right)
$$

Since $H$ is a surjective submersion we can choose a lift $\eta_{n}:\left[-T_{n}, T_{n}\right] \rightarrow \mathcal{M}$ of $\gamma_{n}$ with $\eta_{n}^{\prime} \perp \operatorname{ker} H_{*}$. Then there exists a constant $C_{H}<\infty$, depending only on $H$, such that $\frac{1}{\left\|H_{*}\right\|_{\infty}} \leq\left|\eta_{n}^{\prime}\right| \leq C_{H}$, where $\left\|H_{*}\right\|_{\infty}$ denotes the $C^{0}$-norm of $H_{*}$. Since $\mathcal{M}$ is compact we can assume that

$$
\frac{1}{2 T_{n}}\left(\eta_{n}^{\prime}\right)_{\sharp}\left(\left.\mathcal{L}^{1}\right|_{\left[-T_{n}, T_{n}\right]}\right) \stackrel{*}{\rightarrow} \mu \in C^{0}(T \mathcal{M})^{\prime} .
$$

By the above bound on $\left|\eta_{n}^{\prime}\right|$ we have $\mathcal{Z} \cap \operatorname{supp} \mu=\emptyset$. Let $v \in \operatorname{supp} \mu$ and define $x:=\pi_{T \mathcal{M}}(v)$. By perturbing the map $H$ around $\{x\} \times S^{1}$, we can assume that $r k\left\{H_{*}((v, 0)), H_{*}\left(\partial_{\varphi}\right)\right\}=2$ for all $\varphi \in S^{1}$, i.e. $H_{*}\left(X_{H}\right)_{(v, \varphi)} \neq 0$. Choose $\varepsilon_{0}>0$ such that $\left.H_{*}\left(X_{H}\right)\right|_{B_{\varepsilon_{0}}(v) \times S^{1}}$ is future pointing lightlike and $\delta>0$ such that $\mu\left(B_{\varepsilon_{0}}(v)\right) \geq 2 \delta$. Then we have

$$
\frac{1}{2 T_{n}}\left(\eta_{n}^{\prime}\right)_{\sharp}\left(\left.\mathcal{L}^{1}\right|_{\left[-T_{n}, T_{n}\right]}\right)\left(B_{\varepsilon_{0}}(v)\right) \geq \delta
$$

for sufficiently large $n \in \mathbb{N}$.

Let $(t, \varphi)$ be the natural coordinates on $\left[-T_{n}, T_{n}\right] \times S^{1}$. By construction $X_{H}$ induces a vector field $X_{n}$ on $\left[-T_{n}, T_{n}\right] \times S^{1}$ via the condition $\left(\eta_{n}, \text { id }\right)_{*}\left(X_{n}\right):=X_{H}$. Necessary properties of $X_{n}$ are

$$
\left.d \varphi\left(X_{n}\right)\right|_{\left[-T_{n}, T_{n}\right] \times\{0\}}=0 \text { and } d t\left(X_{n}\right)>\varepsilon_{1}
$$

for some $\varepsilon_{1}>0$. Next consider $\varepsilon_{2}>0$ such that

$$
\left(\eta_{n}, \mathrm{id}\right)\left(\left[-T_{n}, T_{n}\right] \times\left[-\varepsilon_{2}, \varepsilon_{2}\right]\right) \subseteq B_{\varepsilon}\left(H_{*}^{-1}\left(\operatorname{Light}(M, g) \cap T^{1} M\right) \cap\left(\operatorname{ker} H_{*}\right)^{\perp}\right)
$$


Note that we can choose $\varepsilon_{2}$ independent of $n$, since $\mathcal{M}$ is compact. Then the restriction $\left.X_{n}\right|_{\left[-T_{n}, T_{n}\right] \times\left[-\varepsilon_{2}, \varepsilon_{2}\right]}$ is $L$-Lipschitz, since by construction $\eta_{n}^{\prime} \perp$ ker $H_{*}$ and $H_{*}\left(\eta_{n}^{\prime}\right)=\gamma_{n}^{\prime} \in \operatorname{Light}(M, g) \cap T^{1} M$. Next define the vector field

$$
Y_{n}:\left[-T_{n}, T_{n}\right] \times S^{1} \rightarrow T\left(\left[-T_{n}, T_{n}\right] \times S^{1}\right),(t, \varphi) \mapsto X_{n}+\frac{1}{\sqrt{|t|+1}} \partial_{\varphi} .
$$

Note that $\left(H \circ\left(\eta_{n}, \mathrm{id}\right)\right)_{*}\left(Y_{n}\right)$ is always future pointing timelike in $(M, g)$. Consider a maximal solution $\xi_{n}:\left[\alpha_{n}, \omega_{n}\right] \rightarrow\left[-T_{n}, T_{n}\right] \times S^{1}$ of $\xi_{n}^{\prime}=Y_{n}\left(\xi_{n}\right)$ with initial value $(0,0) \in\left[-T_{n}, T_{n}\right] \times S^{1}$. With the definition of $Y_{n}$ we have $-\alpha_{n}, \omega_{n} \in\left[\varepsilon_{1} T_{n}, T_{n}\right]$ and therefore $-\alpha_{n}, \omega_{n} \geq \frac{1}{\varepsilon_{2}}$ if $T_{n} \geq \frac{1}{\varepsilon_{1} \varepsilon_{2}}$. Next consider positive integers $k$ such that $\frac{1}{\varepsilon_{2}} \leq \tau_{k}^{\omega}:=k(k+1) / 2 \leq \omega_{n}$ and $-\frac{1}{\varepsilon_{2}} \geq \tau_{k}^{\alpha}:=-k(k+1) / 2 \geq \alpha_{n}$. We have $d \varphi\left(\xi_{n}^{\prime}(\tau)\right) \leq\left(L+\sqrt{\frac{2}{\varepsilon_{1}}}\right) \frac{1}{k}$ for $|\tau| \geq \tau_{k}^{\omega}=-\tau_{k}^{\alpha}, \varphi\left(\xi_{n}(\tau)\right) \in[-1 / k, 1 / k]$, and consequently

$$
\int_{\tau_{k}^{\omega}}^{\tau_{k+1}^{\omega}} d \varphi\left(\xi_{n}^{\prime}\right) d \tau, \int_{\tau_{k+1}^{\alpha}}^{\tau_{k}^{\alpha}} d \varphi\left(\xi_{n}^{\prime}\right) d \tau \leq 2\left(L+\frac{1}{\sqrt{\varepsilon_{1}}}\right)+1 .
$$

Therefore there exists $C_{1}<\infty$ such that

$$
\int_{\alpha_{n}}^{\omega_{n}} d \varphi\left(\xi_{n}^{\prime}\right) d \tau \leq C_{1} \sqrt{\omega_{n}-\alpha_{n}}
$$

for $n$ sufficiently large. Note that the integral $\int_{-1 / \varepsilon_{2}}^{1 / \varepsilon_{2}} d \varphi\left(\xi^{\prime}\right)$ is uniformly bounded. Set

$$
\zeta_{n}:=H \circ\left(\eta_{n}, \mathrm{id}\right) \circ \xi_{n}:\left[\alpha_{n}, \omega_{n}\right] \rightarrow M .
$$

By construction $\zeta_{n}$ is future pointing timelike. Next we estimate the $g$-length of $\zeta_{n}$. Since $H_{*}\left(\partial_{\varphi}\right)$ is future pointing timelike, $H_{*}\left(X_{H}\right)$ is future pointing or vanishing and $\zeta_{n}^{\prime}(\tau)=H_{*}\left(\partial_{\varphi}\right)+\frac{1}{\sqrt{\left|t\left(\xi_{n}(\tau)\right)\right|+1}} H_{*}\left(X_{H}\right)$, we have $\left(\left|t\left(\xi_{n}(\tau)\right)\right| \leq|\tau|\right)$

$$
L^{g}\left(\zeta_{n}\right) \geq \int_{\alpha_{n}}^{\omega_{n}} \frac{\sqrt{\left.2\left|H^{*} g\left(\partial_{\varphi}, X_{H}\right)\right|\right)}}{\left(\left|t\left(\xi_{n}(\tau)\right)\right|+1\right)^{1 / 4}} d \tau \geq \int_{\alpha_{n}}^{\omega_{n}} \frac{\sqrt{2\left|H^{*} g\left(\partial_{\varphi}, X_{H}\right)\right|}}{(|\tau|+1)^{1 / 4}} d \tau .
$$

Recall that $\left.H_{*}\left(X_{H}\right)\right|_{B_{\varepsilon_{0}}(v) \times S^{1}}$ is future pointing lightlike, i.e. $H^{*} g\left(\partial_{\varphi}, X_{H}\right) \neq 0$ on $B_{\varepsilon_{0}}(v) \times S^{1}$. By decreasing $\varepsilon_{0}$ (and with it $\delta$ ) we can assume that there exists $\varepsilon_{3}>0$ such that $\left|g\left(H_{*} \partial_{\varphi}, H_{*} X_{H}\right)\right|\left(\zeta_{n}(\tau)\right) \geq \varepsilon_{3}$ whenever $\zeta_{n}(\tau) \in H\left(B_{\varepsilon_{0}}(\pi(v)) \times S^{1}\right)$. The average amount of time that $\zeta_{n}$ intersects $H\left(B_{\varepsilon_{0}}(\pi(v)) \times S^{1}\right)$ is bounded from below by $\varepsilon_{1} \delta$. Therefore we obtain

$$
L^{g}\left(\zeta_{n}\right) \geq \frac{\sqrt{\varepsilon_{3}}}{2} \varepsilon_{1} \delta\left(1-\frac{\varepsilon_{1} \delta}{2}\right)^{3 / 4}\left(\omega_{n}-\alpha_{n}\right)^{3 / 4}
$$

for $\varepsilon_{1} \delta\left(\omega_{n}-\alpha_{n}\right) \geq 2$.

Since $h_{H} \in \mathfrak{T}^{\circ}$, by our assumption on $\gamma_{n}$ and (4), we obtain

$$
\operatorname{dist}_{\|.\|}\left(\zeta_{n}\left(\omega_{n}\right)-\zeta_{n}\left(\alpha_{n}\right), \operatorname{pos}\left\{h_{H}, h\right\}\right) \leq \operatorname{err}\left(g, g_{R}\right) .
$$


We extend the curves $\zeta_{n}$, using [20, Proposition 27], by uniformly bounded arcs to future pointing curves $\bar{\zeta}_{n}$ with $h_{n}:=\rho\left(\bar{\zeta}_{n}\right) \in \operatorname{pos}\left\{h_{H}, h\right\}$. Equation (4) shows that

$$
\operatorname{dist}_{\|\cdot\|}\left(h_{n}, \operatorname{pos}\{h\}\right) \leq \frac{C_{1}}{\sqrt{\omega_{n}-\alpha_{n}}} .
$$

By [20, Theorem 11(ii)] there exists $\lambda>0$ such that $\lim _{n \rightarrow \infty} h_{n}=\lambda h$. Since $\mathfrak{l}\left(h_{n}\right) \geq \frac{L^{g}\left(\bar{\zeta}_{n}\right)}{2\left(\omega_{n}-\alpha_{n}\right)}$, for sufficiently large $n$, we obtain, using (5),

$$
\mathfrak{l}\left(h_{n}\right) \geq \frac{\varepsilon_{4}}{\left(\omega_{n}-\alpha_{n}\right)^{1 / 4}}
$$

for $n$ sufficiently large and some $\varepsilon_{4}>0$, independent of $n$. But then for sufficiently large $n$ there exists $\varepsilon_{5}>0$ with

$$
\mathfrak{l}\left(h_{n}\right) \geq \varepsilon_{5} \sqrt{\operatorname{dist}_{\|.\|}\left(h_{n}, \operatorname{pos}\{h\}\right)} .
$$

For any support function $\alpha \in \mathfrak{T}^{*}$ of $\mathfrak{l}$ we have $\mathfrak{l}(h) \leq \alpha(h)$. If we assume $\alpha \in \partial \mathfrak{T}^{*}$, there exists $h_{\alpha} \in \partial \mathfrak{T} \backslash\{0\}$ with $\alpha\left(h_{\alpha}\right)=0$. Consequently, we would have

$$
\mathfrak{l}(h) \leq\|\alpha\|^{*} \operatorname{dist}_{\|\cdot\|}\left(h, \operatorname{pos}\left\{h_{\alpha}\right\}\right)
$$

for all $h \in \mathfrak{T}$. This contradicts equation (6) for a suitable sequence $\left\{h_{n, \alpha}\right\}_{n \in \mathbb{N}}$.

For the next proofs we need to recall the definition of calibrations in Lorentzian geometry from [21]. Denote with

$$
\mathfrak{l}^{*}: \mathfrak{T}^{*} \rightarrow \mathbb{R}, \quad \mathfrak{l}^{*}(\alpha)=\min \{\alpha(h) \mid \mathfrak{l}(h)=1\}
$$

the dual function of the stable time separation.

Let $\alpha \in H^{1}(M, \mathbb{R})$. We call a function $\tau: \bar{M} \rightarrow \mathbb{R} \alpha$-equivariant if $\tau(x+k)=$ $\tau(x)+\alpha(k)$ for all $x \in \bar{M}$ and $k \in H_{1}(M, \mathbb{Z})$. Here $x \mapsto x+k$ denotes the Deck transformation induced by $k \in H_{1}(M, \mathbb{Z})$ on the Abelian cover.

Definition 3.5. [ [21]] Let $\alpha \in\left(\mathfrak{T}^{*}\right)^{\circ}$. An $\alpha$-equivariant and Lipschitz continuous function $\tau: \bar{M} \rightarrow \mathbb{R}$ is a calibration representing $\alpha$ if

$$
\tau(\bar{q})-\tau(\bar{p}) \geq \mathfrak{l}^{*}(\alpha) d(\bar{p}, \bar{q})
$$

for all $\bar{p}, \bar{q} \in \bar{M}$ which are endpoints of a future pointing curve.

For $\alpha \in \mathfrak{T}^{*}$ define $\mathfrak{M}_{\alpha}$ to be the set of invariant measures $\mu$ that maximize

$$
\mu \mapsto \digamma^{*}(\alpha) \int_{T^{1} M} \sqrt{|g(v, v)|} d \mu(v)-\langle\alpha, \rho(\mu)\rangle .
$$

Set supp $\mathfrak{M}_{\alpha}:=\cup_{\mu \in \mathfrak{M}_{\alpha}} \operatorname{supp} \mu$. 
Proof of Proposition 2.13. Let $\varepsilon>0$ be given. Assume that there exists a sequence of maximal measures $\mu_{n}$ with $\rho\left(\mu_{n}\right) \in \mathfrak{T}_{\varepsilon}$ and $\operatorname{dist}\left(\operatorname{supp} \mu_{n}, \operatorname{Light}(M, g)\right) \rightarrow 0$ for $n \rightarrow \infty$. We can assume that the $\mu_{n}$ 's are all probability measures. Choose a weakly converging subsequence $\mu_{n_{k}}$ with weak limit $\mu$. Denote with $h \in \mathfrak{T}_{\varepsilon} \backslash\{0\}$ the rotation class of $\mu$. Note that $\mu$ is maximal and $\operatorname{dist}(\operatorname{supp} \mu, \operatorname{Light}(M, g))=0$. Consider any support function $\alpha$ of $\mathfrak{l}$ at $h$. By Theorem 2.11 we have $\alpha \in\left(\mathfrak{T}^{*}\right)^{\circ}$. Since $\mu \in \mathfrak{M}_{\alpha}$, [21, Proposition 2.20] implies that any maximizer $\gamma$ with tangents in supp $\mu$ is calibrated by any calibration representing $\alpha$. But then the conclusion $\operatorname{dist}(\operatorname{supp} \mu, \operatorname{Light}(M,[g]))=0$ contradicts [21, Theorem 2.21].

Proof of Theorem 2.15. Fix $\alpha \in\left(\mathfrak{T}^{*}\right)^{\circ}$ and consider $D:=\alpha^{-1}(1) \cap \mathfrak{T}$. Then, by Theorem 2.11, $\mathfrak{l}_{D}$ is a concave function and every support function $\beta: \alpha^{-1}(1) \rightarrow$ $\mathbb{R}$ of $\left.\mathfrak{l}\right|_{D}$ satisfies $\beta^{-1}(0) \cap D=\emptyset$. This follows from the fact that any affine function on $\alpha^{-1}(1)$ has a unique linear extension to $H_{1}(M, \mathbb{R})$, and the linear extensions of support functions of $\mathfrak{l}_{D}$ are support functions of $\mathfrak{l}$. Next consider the compact convex body

$$
\mathcal{K}:=\{(h, t) \mid h \in D, 0 \leq t \leq \mathfrak{l}(h)\}
$$

in $\alpha^{-1}(1) \times \mathbb{R}$. Recall that any point $(h, t) \in \mathcal{K}$ is the convex combination of at most $b$ extremal points of $\mathcal{K}$. Thus for every $(h, t) \in \mathcal{K}$ we can choose extremal points $\left(h_{i}, t_{i}\right)$ and $\lambda_{i} \in(0,1]$ such that $(h, t)=\sum_{i=1}^{b} \lambda_{i}\left(h_{i}, t_{i}\right)$. In the case that $t=\mathfrak{l}(h)$, we obtain that $\left.\mathfrak{l}\right|_{\operatorname{conv}}\left\{h_{i}\right\}_{1 \leq i \leq b}$ is affine and $t_{i}=\mathfrak{l}\left(h_{i}\right)$ for all $i$, since $\mathfrak{l}$ is concave and $(h, \mathfrak{l}(h))$ belongs to the relative interior relint $\left(\operatorname{conv}\left\{h_{i}\right\}_{1 \leq i \leq b}\right)$. Choose any support function $\beta$ of $\left.\mathfrak{l}\right|_{D}$ at $h \in \operatorname{relint}(D)$. Then we have $\beta \equiv \mathfrak{l}$ on $\operatorname{conv}\left\{h_{i}\right\}_{1 \leq i \leq b}$. If there exists $1 \leq i_{0} \leq b$ with $\mathfrak{l}\left(h_{i_{0}}\right)=0$, we obtain $\beta\left(h_{i_{0}}\right)=0$ and a contradiction to our observation that $\beta^{-1}(0) \cap D=\emptyset$ for all support functions $\beta$ of $\left.\mathfrak{l}\right|_{D}$ follows. Therefore any point $(h, \mathfrak{l}(h)) \in \mathcal{K}$ with $h \in \operatorname{relint}(D)$ is the convex combination of extremal points $\left(h^{\prime}, \mathfrak{l}\left(h^{\prime}\right)\right)$ of $\mathcal{K}$ with $\mathfrak{l}\left(h^{\prime}\right)>0$.

Choose homology classes $h_{j} \in \operatorname{relint}(D)$ for $1 \leq j \leq b$ such that $\left\{h_{j}\right\}_{1 \leq j \leq b}$ is a basis of $H_{1}(M, \mathbb{R})$ and support functions $\beta_{j}$ of $\mathfrak{l}_{D}$ at $h_{j}$. Next choose for every $j$ a set of extremal points $\left\{\left(h_{j, i}, \mathfrak{l}\left(h_{j, i}\right)\right)\right\}_{1 \leq i \leq b_{j}}$ of $\mathcal{K}$ and $\lambda^{j, i} \in(0,1]$ such that

$$
\sum_{i} \lambda^{j, i}\left(h_{j, i}, \mathfrak{l}\left(h_{j, i}\right)\right)=\left(h_{j}, \mathfrak{l}\left(h_{j}\right)\right) .
$$

We have seen that every $\beta_{j}$ is a support function of $\mathfrak{l |}_{D}$ at $h_{j, i}$ for every $1 \leq i \leq b_{j}$ as well. Choose a basis $\left\{h_{k}^{\prime}\right\}_{1 \leq k \leq b} \subseteq\left\{h_{j, i}\right\}_{1 \leq j \leq b, 1 \leq i \leq b_{j}}$ of $H_{1}(M, \mathbb{R})$. Fix $1 \leq k \leq b$. Like in the proof of [21, Theorem 2.10] we can consider $\lambda_{k}>0$ maximal among all $\lambda>0$ with

$$
\rho(\mu)=\lambda h_{k}^{\prime} \quad \text { and } \quad \int_{\operatorname{Time}(M, g)} \sqrt{|g(v, v)|} d \mu(v)=\lambda \mathfrak{l}\left(h_{k}^{\prime}\right)
$$

for some invariant probability measure $\mu$. The preimage of $\lambda_{k}\left(h_{k}^{\prime}, \mathfrak{l}\left(h_{k}^{\prime}\right)\right)$ under the map

$$
\mu \mapsto\left(\rho(\mu), \int_{\text {Time }(M, g)} \sqrt{|g(v, v)|} d \mu(v)\right)
$$


is compact and convex in the space of invariant probability measures. Therefore it contains extremal points by the Theorem of Krein-Milman. Every extremal point of this preimage is an extremal point of the space of invariant probability measures. Since all measures in the preimage of $\lambda_{k}\left(h_{k}^{\prime}, \mathfrak{l}\left(h_{k}^{\prime}\right)\right)$ are maximal, the extremal points are maximal ergodic measures. Choose a maximal ergodic measure $\mu_{k}$ in the preimage. The unique linear extension $\alpha_{k} \in H^{1}(M, \mathbb{R})$ of $\beta_{k}$ is a support function of $\mathfrak{l}$ and therefore we have $\alpha_{k} \in\left(\mathfrak{T}^{*}\right)^{\circ}$. By our choice we have $\mu_{k} \in \mathfrak{M}_{\alpha_{k}}$. Then with [21, Theorem 2.21] we get supp $\mu_{k} \subseteq \operatorname{Time}(M, g)$, i.e. there exist $\varepsilon_{k}>0$ with $\operatorname{dist}\left(\operatorname{supp} \mu_{k}, \operatorname{Light}(M, g)\right) \geq \varepsilon_{k}$. Setting $\varepsilon:=\min \left\{\varepsilon_{k}\right\}$, the claim follows.

Proof of Corollary 2.16. The proof of Theorem 2.15 especially shows that for class $\mathrm{A}_{1}$ spacetimes with $\mathfrak{l}=0$ somewhere on $\partial \mathfrak{T} \backslash\{0\}$ (l can only vanish on $\left.\partial \mathfrak{T}\right)$, there exist infinitely many ergodic maximal measures $\mu$ with supp $\mu \subseteq \operatorname{Time}(M, g)$. This follows from the observation that if $\mathfrak{l}$ vanishes somewhere on $\partial \mathfrak{T} \backslash\{0\}$, the number of extremal points of $\mathcal{K}$ cannot be finite.

\subsection{Lipschitz continuity of the time separation}

Proposition 3.6. Let $(M, g)$ be of class $A_{1}$. Then for all $\varepsilon>0$ there exist $\delta>0$ and $K<\infty$ such that

$$
\dot{\gamma}(t) \in \operatorname{Time}(M,[g])^{\delta}
$$

forallmaximizers $\gamma:[a, b] \rightarrow M$ with $\gamma(b)-\gamma(a) \in \mathfrak{T}_{\varepsilon} \backslash B_{K}(0)$ and all $t \in[a, b]$.

We obtain the following immediate corollary.

Corollary 3.7. Let $(M, g)$ be of class $A_{1}$. For every $\varepsilon>0$ there exists $K=K(\varepsilon)<$ $\infty$ such that for every sequence of maximizers $\left\{\gamma_{n}\right\}_{n \in \mathbb{N}}$ with $L^{g_{R}}\left(\gamma_{n}\right) \geq K$ and $\rho\left(\gamma_{n}\right) \in \mathfrak{T}_{\varepsilon}$, any limit curve of $\left\{\gamma_{n}\right\}_{n \in \mathbb{N}}$ is timelike.

We will need the following theorem from [4]:

Theorem 3.8. The stable norm $\|$.$\| on H_{1}(M, \mathbb{R})$ is the unique norm such that there exists a constant $K_{1}<\infty$ with

$$
|\operatorname{dist}(\bar{x}, \bar{y})-\|\bar{y}-\bar{x}\|| \leq K_{1}
$$

for any pair $\bar{x}, \bar{y} \in \bar{M}$.

Proof of Proposition 3.6. Choose using [20, Proposition 27] $K(\varepsilon)<\infty$ such that the assumptions $y-x \in \mathfrak{T}_{\varepsilon}$ and $\|y-x\| \geq K(\varepsilon)$ imply that $x$ and $y$ are connected by a future pointing timelike curve. The idea is to confirm the existence of $\delta(\varepsilon)>0$ such that for all $x, y \in \bar{M}$ with $\|y-x\| \geq K(\varepsilon)$ and $y-x \in \mathfrak{T}_{\varepsilon}$, any future pointing maximizer $\gamma:[0, T] \rightarrow \bar{M}$ from $x$ to $y$ satisfies

$$
\dot{\gamma}(t) \in \operatorname{Time}(M,[g])^{\delta(\varepsilon)}
$$

for all $t \in[0, T]$. Assume the contrary, i.e. there exist a sequence of pairs $\left(x_{n}, y_{n}\right) \in$ $\bar{M} \times \bar{M}$ with $y_{n}-x_{n} \in \mathfrak{T}_{\varepsilon}$, maximizers $\gamma_{n}:\left[0, T_{n}\right] \rightarrow \bar{M}$ connecting $x_{n}$ and $y_{n}$ 
and parameter values $t_{n} \in\left[0, T_{n}\right]$ with $\dot{\gamma}_{n}\left(t_{n}\right) \notin \operatorname{Time}(M,[g])^{1 / n}$. The sequence $y_{n}-x_{n}$ cannot have any accumulation points by the choice of $K(\varepsilon)$. If there exist points of accumulation $x, y$, the curves $\gamma_{n}$ will accumulate towards a maximizer with vanishing length. Then since $\mathfrak{T}_{\varepsilon}$ is closed, there exists a lightlike maximizer connecting points $x$ and $y$ with $y-x \in \mathfrak{T}_{\varepsilon}$. This contradicts the choice of $K(\varepsilon)$. Consequently the sequence $\left\{y_{n}-x_{n}\right\}_{n \in \mathbb{N}}$ must be unbounded.

Since $\operatorname{Light}(M,[g]) \cap T^{1} M$ is $\Phi$-invariant and $\Phi$ is complete as well as continuous, there exists a sequence $0<a_{n} \rightarrow \infty$ such that $L^{g}\left(\left.\gamma_{n}\right|_{\left[t_{n}-a_{n}, t_{n}\right]}\right) \leq 1$ and

$$
\left\|\gamma_{n}\left(t_{n}\right)-\gamma_{n}\left(t_{n}-a_{n}\right)\right\| \leq \frac{\varepsilon}{4}\left\|\gamma_{n}\left(T_{n}\right)-\gamma_{n}(0)\right\| \leq \frac{1}{4} \operatorname{dist}_{\|.\|}\left(\gamma_{n}\left(T_{n}\right)-\gamma_{n}(0), \partial \mathfrak{T}\right)
$$

for all $n \in \mathbb{N}$. This implies

$$
\left[\gamma_{n}\left(t_{n}-a_{n}\right)-\gamma_{n}(0)\right]+\left[\gamma_{n}\left(T_{n}\right)-\gamma_{n}\left(t_{n}\right)\right]=: v_{n} \in \mathfrak{T}_{\frac{3 \varepsilon}{4}}
$$

Fix a lift $\bar{\gamma}_{n}:\left[0, T_{n}\right] \rightarrow \bar{M}$ of $\gamma_{n}$. With [20, Note 1$]$ there exists a constant $K_{2}<\infty$ and $k_{n} \in H_{1}(M, \mathbb{Z})$ such that

$$
\operatorname{dist}\left(\bar{\gamma}_{n}\left(T_{n}\right), \bar{\gamma}_{n}(0)+k_{n}\right) \leq K_{2}
$$

and $\bar{\gamma}_{n}(0)+k_{n}$ is the endpoint of a future pointing curve starting in $\bar{\gamma}_{n}\left(T_{n}\right)$. Then $\left[\bar{\gamma}_{n}\left(t_{n}-a_{n}\right)+k_{n}\right]-\bar{\gamma}_{n}\left(t_{n}\right) \in \mathfrak{T}_{\varepsilon / 2}$ for sufficiently large $n$. Denote with $L_{\varepsilon / 2}$ the Lipschitz constant of $\left.\mathfrak{l}\right|_{\mathfrak{T}_{\varepsilon / 2}}$. We obtain with Theorem 2.10 and Theorem 3.8

$$
\begin{aligned}
& \mathfrak{l}\left(v_{n}\right)+\mathfrak{l}\left(\gamma_{n}\left(t_{n}\right)-\gamma_{n}\left(t_{n}-a_{n}\right)\right) \leq \mathfrak{l}\left(\gamma_{n}\left(T_{n}\right)-\gamma_{n}(0)\right) \leq d\left(\bar{\gamma}_{n}(0), \bar{\gamma}_{n}\left(T_{n}\right)\right)+\bar{C}(\varepsilon) \\
& \quad=d\left(\bar{\gamma}_{n}(0), \bar{\gamma}_{n}\left(t_{n}-a_{n}\right)\right)+d\left(\bar{\gamma}_{n}\left(t_{n}-a_{n}\right), \bar{\gamma}_{n}\left(t_{n}\right)\right)+d\left(\bar{\gamma}_{n}\left(t_{n}\right), \bar{\gamma}_{n}\left(T_{n}\right)\right)+\bar{C}(\varepsilon) \\
& \quad \leq d\left(\bar{\gamma}_{n}\left(t_{n}\right), \bar{\gamma}_{n}\left(t_{n}-a_{n}\right)+k_{n}\right)+d\left(\bar{\gamma}_{n}\left(t_{n}-a_{n}\right), \bar{\gamma}_{n}\left(t_{n}\right)\right)+\bar{C}(\varepsilon) \\
& \quad \leq \mathfrak{l}\left(\left[\bar{\gamma}_{n}\left(t_{n}-a_{n}\right)+k_{n}\right]-\bar{\gamma}_{n}\left(t_{n}\right)\right)+1+2 \bar{C}(\varepsilon / 2) \\
& \quad \leq \mathfrak{l}\left(v_{n}\right)+L_{\varepsilon / 2}\left(K_{2}+\mathrm{K}_{1}\right)+1+2 \bar{C}(\varepsilon / 2) .
\end{aligned}
$$

Consequently

$$
\mathfrak{l}\left(\gamma_{n}\left(t_{n}\right)-\gamma_{n}\left(t_{n}-a_{n}\right)\right) \leq L_{\varepsilon / 2}\left(K_{2}+\mathrm{K}_{1}\right)+1+2 \bar{C}(\varepsilon)=: C_{1}(\varepsilon) .
$$

From $a_{n} \rightarrow \infty$ and $\mathfrak{l}_{\mathfrak{T}^{\circ}}>0$ we obtain

$$
w_{n}:=\gamma_{n}\left(t_{n}\right)-\gamma_{n}\left(t_{n}-a_{n}\right) \notin \mathfrak{T}_{\varepsilon / 2}
$$

for $n$ sufficiently large. Therefore the homology classes $v_{n}+w_{n}$ and $v_{n}$ are linearly independent and we can define an "almost support" function $\alpha_{n}$ of $\mathfrak{l}$ as follows. Set $\alpha_{n}\left(v_{n}\right):=\mathfrak{l}\left(v_{n}\right)$ and $\alpha_{n}\left(v_{n}+w_{n}\right):=\mathfrak{l}\left(v_{n}+w_{n}\right)$. This defines a unique linear function $\alpha_{n}$ on $\operatorname{span}\left\{v_{n}, w_{n}\right\}$. With [20, Proposition 8] there exists $K_{3}<\infty$ such that for any $\lambda \in[0,1]$ we have

$$
\begin{aligned}
\mathfrak{l}\left(v_{n}+w_{n}\right) & \geq \mathfrak{l}\left(v_{n}+\lambda w_{n}\right)-L_{\varepsilon / 2} \operatorname{dist}_{\|\cdot\|}\left((1-\lambda) w_{n}, \mathfrak{T}\right) \\
& \geq \mathfrak{l}\left(v_{n}+\lambda w_{n}\right)-L_{\varepsilon / 2} K_{3}
\end{aligned}
$$


and consequently

$$
\mathfrak{l}\left(v_{n}+\lambda w_{n}\right) \leq \mathfrak{l}\left(v_{n}\right)+C_{1}(\varepsilon)+L_{\varepsilon / 2} K_{3}=: \mathfrak{l}\left(v_{n}\right)+C_{2}(\varepsilon) .
$$

With the definition of $\alpha_{n}$ and $\mathfrak{l}\left(v_{n}\right) \leq \mathfrak{l}\left(v_{n}+w_{n}\right)$ we obtain

$$
\alpha_{n}\left|\operatorname{conv}\left\{v_{n}, v_{n}+w_{n}\right\} \geq \mathfrak{l}\right| \operatorname{conv}\left\{v_{n}, v_{n}+w_{n}\right\}-C_{2}(\varepsilon)
$$

and therefore

$$
\alpha_{n}(h) \geq \mathfrak{l}(h)-\|h\| \frac{C_{2}(\varepsilon)}{\min \left\{\left\|v_{n}+\lambda w_{n}\right\| \mid \lambda \in[0,1]\right\}}
$$

for all $h \in \operatorname{pos}\left\{v_{n}, v_{n}+w_{n}\right\}$. Now the concavity of $\mathfrak{l}$ and the definition of $\alpha_{n}$ imply (7) for all $h \in \operatorname{span}\left\{v_{n}, w_{n}\right\} \cap \mathfrak{T}$. Choose, using the Hahn-Banach Theorem, an extension $\beta_{n}: H_{1}(M, \mathbb{R}) \rightarrow \mathbb{R}$ of $\alpha_{n}$ such that

$$
\beta_{n} \geq \mathfrak{l}-\|\cdot\| \frac{C_{2}(\varepsilon)}{\min \left\{\left\|v_{n}+\lambda w_{n}\right\| \mid \lambda \in[0,1]\right\}} .
$$

Since $v_{n}+w_{n} \in \mathfrak{T}_{\varepsilon}$ and $\alpha_{n}\left(v_{n}+w_{n}\right)=\mathfrak{l}\left(v_{n}+w_{n}\right)>0$ uniformly in $n$, we obtain that $\|\beta\|^{*}$ is bounded away from 0 and $\infty$, uniformly in $n$.

Choose converging subsequences $\beta_{n_{k}} \rightarrow \beta \in H^{1}(M, \mathbb{R}) \backslash\{0\}, v_{n_{k}} /\left\|v_{n_{k}}\right\| \rightarrow$ $v \in \mathfrak{T}_{\varepsilon / 2}$ and $w_{n_{k}} /\left\|w_{n_{k}}\right\| \rightarrow w \in \mathfrak{T}$. Since $\mathfrak{T}$ contains no linear subspaces, we have $\min \left\{\left\|v_{n}+\lambda w_{n}\right\| \mid \lambda \in[0,1]\right\} \rightarrow \infty$ for $n \rightarrow \infty$. By continuity of $\mathfrak{l}$ on $\mathfrak{T}^{\circ}$ we have $\beta(v)=\mathfrak{l}(v)$ and therefore $\beta \in \mathfrak{T}^{*}$. Note that we have

$$
\beta_{n}\left(v_{n}+w_{n}\right)=\mathfrak{l}\left(v_{n}+w_{n}\right) \leq \mathfrak{l}\left(v_{n}\right)+C_{1}(\varepsilon)=\beta_{n}\left(v_{n}\right)+C_{1}(\varepsilon)
$$

and therefore $\beta_{n}\left(w_{n}\right) \leq C_{1}(\varepsilon)$. Thus we get $\beta(w)=0$ and a contradiction to Theorem 2.11 .

Proof of Theorem 2.17. The proof is similar to the one of [6, Theorem 3.7]. We use the following lemma proved in the appendix of [5].

Lemma. Let $U$ be an open convex domain in $\mathbb{R}^{n}$ and $f: U \rightarrow \mathbb{R}$ a continuous function. Assume that for any $q \in U$ there is a smooth lower support function $f_{q}$ at $q$ such that $\left|\left(f_{q}\right)_{* q}\right| \leq L$. Then $f$ is Lipschitz with Lipschitz constant L.

For a given $\varepsilon>0$ choose $K(\varepsilon)<\infty, \delta(\varepsilon)>0$ as in Proposition 3.6 and let $x, y \in$ $\bar{M}$ with $y-x \in \mathfrak{T}_{\varepsilon} \backslash B_{2 K(\varepsilon)}(0)$. Further choose a convex normal neighborhood $V$ of $x$ such that $y-z \in \mathfrak{T}_{\varepsilon} \backslash B_{K(\varepsilon)}(0)$ for all $z \in V$. Next choose a maximizer $\gamma$ : $[0, T] \rightarrow \bar{M}$ connecting $x$ with $y$ and $t>0$ such that $\operatorname{dist}(x, \gamma(t)) \geq \operatorname{inj}(\bar{M}, \bar{g}) / 2$. By Theorem 2.11 there exists $\eta=\eta(\varepsilon)>0$, independent of $x$ and $y$, such that

$$
-\left(\exp _{\gamma(t)}^{\bar{g}}\right)^{-1}(z) \in \operatorname{Time}(\bar{M}, \bar{g})_{\gamma(t)}^{\delta(\varepsilon)}
$$

for all $z \in B_{\eta}(x)$. Consider on $B_{\eta}(x)$ the function $f_{x}(z):=d_{V}(z, \gamma(t))+$ $d(\gamma(t), y)$, where $d_{V}$ is the local time separation of $\left(V,\left.\bar{g}\right|_{V}\right)$. Note that $f_{x}$ is smooth on $B_{\eta}(x)$ with bounded differential by Theorem 2.11 and

$$
d_{V}(z, \gamma(t))=\sqrt{\left|g\left(\exp ^{-1}(z), \exp ^{-1}(z)\right)\right|} .
$$


By the reverse triangle inequality, $f_{x}$ is a lower support function of $d(., y)$ at $x$. This establishes the assumption of the lemma and we obtain that the restricted time separation $d(., y)$ is Lipschitz at $x$ with Lipschitz constant depending only on $\varepsilon$. Since the same argument can be applied to $d(x,$.$) , we obtain the Lipschitz$ continuity of the time separation $d$ on $\left\{(x, y) \in \bar{M} \times \bar{M} \mid y-x \in \mathfrak{T}_{\varepsilon} \backslash B_{K(\varepsilon)}(0)\right\}$.

Funding Open Access funding enabled and organized by Projekt DEAL.

Open Access This article is licensed under a Creative Commons Attribution 4.0 International License, which permits use, sharing, adaptation, distribution and reproduction in any medium or format, as long as you give appropriate credit to the original author(s) and the source, provide a link to the Creative Commons licence, and indicate if changes were made. The images or other third party material in this article are included in the article's Creative Commons licence, unless indicated otherwise in a credit line to the material. If material is not included in the article's Creative Commons licence and your intended use is not permitted by statutory regulation or exceeds the permitted use, you will need to obtain permission directly from the copyright holder. To view a copy of this licence, visit http://creativecommons.org/ licenses/by/4.0/.

\section{References}

[1] Bangert, V.: Minimal geodesics. Ergodic Theory Dyn. Syst. 10, 263-286 (1990)

[2] Beem, J.K., Ehrlich, P.E., Easley, K.L.: Global Lorentzian Geometry, 2nd edn. Marcel Dekker Inc., New York (1996)

[3] Bernal, A.N., Sánchez, M.: Globally hyperbolic spacetimes can be defined as 'causal' instead of 'strongly causal'. Class. Quantum Gravity 24, 745-749 (2007)

[4] Burago, D.Yu.: Periodic metrics. Representation theory and dynamical systems. Adv. Soviet Math. 9, 205-210 (1992)

[5] Eschenburg, J.H.: The splitting theorem for space-times with strong energy condition. J. Differ. Geom. 27, 477-491 (1988)

[6] Galloway, G.J., Horta, A.: Regularity of Lorentzian Busemann Functions. Trans. Am. Math. Soc. 348, 2063-2084 (1996)

[7] Gromov M (2007) Metric structures for Riemannian and non-Riemannian spaces, Translated from the French by Sean Michael Bates. With appendices by M. Katz, P. Pansu, and S. Semmes. Edited by J. LaFontaine and P. Pansu, 3rd printing, Modern Birkhäuser Classics, Birkhäuser, Basel (2007)

[8] Hawking, S.W., Ellis, G.F.R.: The Large Scale Structure of Space-Time. Cambridge University Press, London (1973)

[9] Hirsch, M.W.: Differential Topology. Graduate Texts in Mathematics, vol. 33. Springer, New York (1976)

[10] Jin, L., Cui, X.: Global viscosity solutions for eikonal equations on class A Lorentzian 2-tori. Geom. Dedicata 193, 155-192 (2018)

[11] Mather, J.N.: Action minimizing invariant measures for positive definite Lagrangian systems. Math. Z. 207, 169-207 (1991)

[12] Minguzzi, E., Sánchez, M.: The causal hierarchy of spacetimes. In: Baum, H., Alekseevsky, D. (eds.) Recent Developments in Pseudo-Riemannian Geometry, pp. 299358. European Mathematical Society, Zürich (2008) 
[13] O’Neill, B.: Semi-Riemannian Geometry. Academic Press Inc., New York (1983)

[14] Peng, L., Jin, L., Cui, X.: On class A Lorentzian 2-tori with poles I: Closed geodesics pass through poles. Manuscr. Math. 160, 543-559 (2019)

[15] Peng, L., Jin, L., Cui, X.: On class A Lorentzian 2-tori with poles II: Foliations by timelike lines. Differ. Geom. Appl. 65, 16-29 (2019)

[16] Penrose, R.: Techniques of differential topology in relativity. In: Conference Board of the Mathematical Sciences Regional Conference Series in Applied Mathematics, No. 7. SIAM, Philadelphia (1972)

[17] Schelling, E.: Maximale Geodätische auf Lorentzmannigfaltigkeiten. Diplomarbeit, Freiburg (1995)

[18] Suhr, S.: Homologically Maximizing Geodesics in Conformally Flat Tori. AMS/IP Studies in Advanced Mathematics, vol. 49, pp. 125-143. American Mathematical Society, Providence, RI (2011)

[19] Suhr, S.: Closed geodesics in Lorentzian surfaces. Trans. Am. Math. Soc. 365, 14691486 (2013)

[20] Suhr, S.: Class A spacetimes. Geom. Dedicata 160, 91-117 (2012)

[21] Suhr, S.: Aubry-Mather theory for Lorentzian manifolds. J. Fixed Point Theory Appl. 21, 71 (2019)

Publisher's Note Springer Nature remains neutral with regard to jurisdictional claims in published maps and institutional affiliations. 